\title{
Non-linear Supersymmetry for non-Hermitian, non-diagonalizable Hamiltonians: I. General properties
}

\author{
A. A. Andrianov ${ }^{\dagger \star}$, F. Cannata ${ }^{\star}$, A. V. Sokolov ${ }^{\dagger}$ \\ $\dagger$ V.A.Fock Institute of Physics, Sankt-Petersburg State University, \\ * Dipartimento di Fisica and INFN Bologna, Italy
}

\begin{abstract}
We study complex potentials and related non-diagonalizable Hamiltonians with special emphasis on formal definitions of associated functions and Jordan cells. The non-linear SUSY for complex potentials is considered and the theorems characterizing its structure are presented. We define the class of complex potentials invariant under SUSY transformations for (non-)diagonalizable Hamiltonians and formulate several results concerning the properties of associated functions. We comment on the applicability of these results for softly non-Hermitian PT-symmetric Hamiltonians. The role of SUSY (Darboux) transformations in increasing/decreasing of Jordan cells in SUSY partner Hamiltonians is thoroughly analyzed and summarized in the Index Theorem. The properties of nondiagonalizable Hamiltonians as well as the Index Theorem are illustrated in the solvable examples of non-Hermitian reflectionless Hamiltonians. The rigorous proofs are relegated to the Part II of this paper. At last, some peculiarities in resolution of identity for discrete and continuous spectra with a zero-energy bound state at threshold are discussed.
\end{abstract}

\section{Introduction}

Quantum Physics of open systems often deals with incomplete information on the influence of an environment and can be adequately described by non-Hermitian Hamiltonians with a non-positive imaginary part. This kind of effective description has been employed in Condensed Matter, Quantum Optics and Hadronic and Nuclear Physics [1] - 4] for many years. Non-self-adjoint operators were also under mathematical investigations [5, 6, 7] and recently interesting examples of non-Hermitian effective Hamiltonian operators have been found for the quantum many-body equations 8 .

The PT-symmetric Quantum Mechanics proposed in [9, 10] and developed in [10]-14] and its pseudo-Hermitian generalization [15, 16, 17] describes a variety of non-Hermitian Hamiltonians with real spectrum (but not all Hamiltonians with real spectrum are PTsymmetric [18, 19]). There is a progress in understanding some non-Hermitian but PTsymmetric Hamiltonians in terms of Krein spaces [20]. This kind of Quantum Mechanics has attracted much interest as it may open the way to give a solid probabilistic interpretation of non-Hermitian dynamics by means of a positive pseudo-norm [11, 12]. PTsymmetry endows with a physical meaning the energy spectrum of some Hamiltonians formally unbounded from below [9, 10]. The latter possibility for anharmonic oscillators 
with potentials unbounded from below was observed long ago [21, 22, but only recently has been associated with a PT-symmetry [24].

For complex, non-Hermitian potentials the natural spectral decomposition involves biorthogonal states [25]. Moreover the Hamiltonians may not be diagonalizable [26] but can be reduced only to a quasi-diagonal form with a number of Jordan cells [15]. This feature appears at level crossing which, in fact, occurs under specific circumstances in atomic and molecular spectra [26] and Optics [27, 28] as well as in PT-symmetric quantum systems [23, 29, 30]. There are also certain links [31] to the occurrence of non-Hermitian degeneracies for essentially Hermitian Hamiltonians where the description has been developed for complex eigenvalue Gamow states (resonances) unbounded in their asymptotics and, in general, not belonging to the Hilbert space of physical wave functions. On the contrary, in what follows we examine non-Hermitian Hamiltonians with normalizable bound and associated states. The subtleties of biorthogonality (the phenomenon of "self-orthogonality" 28]) in resolution of identity and in definition of quantum averages of observables have been thoroughly analyzed in our paper [32] .

We find it certainly interesting and important to investigate the possible ways for quantum design of such non-Hermitian quantum systems and in particular to extend the methods of non-linear SUSY algebra [33]-64] in order to keep under control the emerging of non-diagonal parts of those systems. In making a link to PT-symmetric systems we restrict ourselves with a soft type of non-Hermiticity when the real part of a potential dominates over the imaginary one at both infinities and asymptotically such a potential remains bounded from below. Respectively the energy spectrum of a related system contains a number of bound states and possibly a continuum part bounded from below. Thus having in mind the SUSY quantum design one can, for instance, think of a chain of complex Hamiltonians produced by Darboux-Crum transformations from a real one as a good representative of the class of softly non-Hermitian systems. The general relations and theorems presented in Sections $2-4$ certainly hold also for PT-symmetric potentials with fixed asymptotics of ratio of imaginary and real part (semihard nonHermiticity), say, for potentials with leading asymptotics $\lambda x^{2 n}(i x)^{\epsilon} ; \lambda>0$ at infinities provided that the boundary conditions for eigenvalue problem do not require to move to complex coordinates [10, 23] (i.e. for $|\epsilon|<1$ ). However the Lemmas and Theorem of Sections 5 and 6 are proven for softly non-Hermitian potentials and we pay hopes to extend them also on semihard non-Hermitian potentials in a nearest future.

We start in Sec.2 with the definitions and a summary of properties of non-Hermitian diagonalizable Hamiltonians and introduce the relevant biorthogonal expansions. Then we consider non-diagonalizable non-Hermitian Hamiltonians with discrete spectrum and finite-size Jordan cells and discuss the choice of the biorthogonal basis with diagonal resolution of identity. The novel result of this section is the proof that a biorthogonal basis always exists which is made of a set of eigenfunctions and associated functions of the initial Hamiltonian and a set of their complex conjugates for the Hermitian conjugated Hamiltonian. Moreover if the Hamiltonian is PT-symmetric and this symmetry is not spontaneously broken on states (eigenvalues are real) then the elements of direct and conjugated bases are related by PT-reflection. In Sec.3 the origin of non-diagonalizable Hamiltonians is clarified to be level confluence.

Non-linear SUSY in QM is summarized and extended to complex potentials in Sec.4 with an emphasis to the possibility of conservation of PT-symmetry. Herein the important theorem on the polynomial structure of SUSY algebra with transposition symmetry as well as the strip-off theorem describing the minimization of the differential order of intertwining operators are adapted to the complex potentials. These theorems involve 
the zero-mode subspaces of supercharge components - intertwining operators and their mapping by Hamiltonians - matrices $S$. They are well compatible with PT-symmetry (if any). The relationship between superpotentials and Wronskians involving associated functions is discussed.

In Sec.5 we present the class of complex potentials invariant under SUSY transformations for (non-)diagonalizable Hamiltonians: this class covers the systems with soft breaking of Hermiticity and essentially real continuum spectrum (if any). For this case we formulate several results characterizing the normalizability of associated functions at $+\infty$ and/or $-\infty$. The necessary conditions for SUSY transformation functions are found to provide a pre-planned Jordan structure of a SUSY partner Hamiltonian. These results allow to unravel the relation between Jordan cells in SUSY partner Hamiltonians the latter being described by the Index Theorem in Sec 6. It represents the main result of the present paper. The Index Theorem relates the dimensions of Jordan cells of super-partner Hamiltonians at any energy level with characteristics of intertwining operator kernels (matrices $S$ )and in fact exhaustively describes the quantum design options for softly non-Hermitian Hamiltonians. Needless to say that the latter theorem is also compatible with PT-symmetry when non-Hermiticity, for instance, is introduced into P-even potentials by shifting of coordinates into complex plane (see examples in [29]) . The illustration of properties of non-diagonalizable Hamiltonians as well as of the Index Theorem is thoroughly performed in Sec. 7 by the solvable example of non-Hermitian reflectionless Hamiltonians originated by SUSY transformations from the free particle Hamiltonian. The arising of non-diagonalizability is illuminated by an exactly solvable system with two coalescing bound states. In Conclusions we outline possible peculiarities of non-Hermitian Hamiltonians with continuous spectrum. The approaching to the continuum threshold yields more subtle problems with normalizable eigen- and associated functions in continuum which may have zero binorm. As a consequence it may cause serious problems with the resolution of identity investigated in detail elsewhere [32].

All the new results on the structure of non-diagonalizable SUSY Hamiltonians presented in this (part of) paper are rigorously proved and justified in the accompanying (second part of) paper [65].

\section{Non-Hermitian diagonalizable vs. non-diago- nalizable Hamiltonians and biorthogonal expansions}

In our paper we deal with complex one-dimensional potentials $V(x) \neq V^{*}(x)$ and respectively with non-Hermitian Hamiltonians $h$ of Schrödinger typ£ 1 , defined on the real axis,

$$
h \equiv-\partial^{2}+V(x)
$$

which are assumed to be symmetric or self-transposed under the ${ }^{t}$ - transposition operation, $h=h^{t}$. The notation $\partial \equiv d / d x$ is employed. Only scalar local potentials will be analyzed which are obviously symmetric under transposition (for some matrix nondiagonalizable problems, see [66, 67]). Taking into account possible applications in PTsymmetric QM we specify complex potentials to give a semihard non-Hermiticity when the real part $\operatorname{Re} V$ is bounded from below and the ratio $\operatorname{Im} V / \operatorname{Re} V$ remains finite and sufficiently small for large $x \rightarrow \pm \infty$. In this case the eigenvalue problem can be safely posed

\footnotetext{
${ }^{1}$ Conventionally the system of units $m=1 / 2, \hbar=c=1$ will be used with dimensionless energies, momenta and coordinates.
} 
keeping the boundary conditions at $x \pm \infty$ on the real axis. Later on, in last two sections we restrict ourselves with softly non-Hermitian potentials with vanishing asymptotic ratios $\operatorname{Im} V / \operatorname{Re} V=o(1)$.

Let us first define a class of one-dimensional non-Hermitian diagonalizable Hamiltonians $h$ with discrete spectrum such that:

a) a biorthogonal system $\left\{\left|\psi_{n}\right\rangle,\left|\tilde{\psi}_{n}\right\rangle\right\}$ exists,

$$
h\left|\psi_{n}\right\rangle=\lambda_{n}\left|\psi_{n}\right\rangle, \quad h^{\dagger}\left|\tilde{\psi}_{n}\right\rangle=\lambda_{n}^{*}\left|\tilde{\psi}_{n}\right\rangle, \quad\left\langle\tilde{\psi}_{n} \mid \psi_{m}\right\rangle=\left\langle\psi_{m} \mid \tilde{\psi}_{n}\right\rangle=\delta_{n m}
$$

b) the complete resolution of identity in terms of these bases and the spectral decomposition of the Hamiltonian hold (in the case of PT-symmetric potentials the necessary conditions for that are formulated in [20]),

$$
I=\sum_{n}\left|\psi_{n}\right\rangle\left\langle\tilde{\psi}_{n}\left|, \quad h=\sum_{n} \lambda_{n}\right| \psi_{n}\right\rangle\left\langle\tilde{\psi}_{n}\right|
$$

In the coordinate representation,

$$
\psi_{n}(x)=\left\langle x \mid \psi_{n}\right\rangle, \quad \tilde{\psi}_{n}(x)=\left\langle x \mid \tilde{\psi}_{n}\right\rangle,
$$

the resolution of identity has the form,

$$
\delta\left(x-x^{\prime}\right)=\left\langle x^{\prime} \mid x\right\rangle=\sum_{n} \psi_{n}\left(x^{\prime}\right) \tilde{\psi}_{n}^{*}(x) .
$$

The differential equations,

$$
h \psi_{n}=\lambda_{n} \psi_{n}, \quad h^{\dagger} \tilde{\psi}_{n}=\lambda_{n}^{*} \tilde{\psi}_{n}
$$

and the fact that there is only one normalizable eigenfunction of $h$ for the eigenvalue $\lambda_{n}$ (up to a constant factor), allow one to conclude that

$$
\tilde{\psi}_{n}^{*}(x) \equiv \alpha_{n} \psi_{n}(x), \quad \alpha_{n}=\text { Const } \neq 0 .
$$

Hence the system $\left\{\left|\psi_{n}\right\rangle,\left|\tilde{\psi}_{n}\right\rangle\right\}$ can be redefined

$$
\left|\psi_{n}\right\rangle \rightarrow \frac{1}{\sqrt{\alpha_{n}}}\left|\psi_{n}\right\rangle, \quad\left|\tilde{\psi}_{n}\right\rangle \rightarrow \sqrt{\alpha_{n}^{*}}\left|\tilde{\psi}_{n}\right\rangle
$$

so that

$$
\tilde{\psi}_{n}^{*}(x) \equiv \psi_{n}(x), \quad \int_{-\infty}^{+\infty} \psi_{n}(x) \psi_{m}(x) d x=\delta_{n m} .
$$

We stress that the non-vanishing binorms in Eq.(9) support the completeness of this basis, i.e. the resolution of identity,

$$
\delta\left(x-x^{\prime}\right)=\sum_{n} \psi_{n}(x) \psi_{n}\left(x^{\prime}\right)
$$

Indeed if some of the states in Eq. (10) were "self-orthogonal" (as it has been accepted in [66]) , i.e. had zero binorms in (9), the would-be unity in (10) would annihilate such states thereby signaling the incompleteness. 
The PT-symmetry of a potential entails a related symmetry of eigenfunctions,

$$
V^{*}(x)=V(-x) \Longrightarrow \tilde{\psi}_{n}(x)=\psi_{n}^{*}(x) \equiv \gamma_{n} \psi_{n}(-x), \quad\left|\gamma_{n}\right|=1 .
$$

when the PT-symmetry is not spontaneously broken, i.e. $\lambda_{n}^{*}=\lambda_{n}$ (further on, for clarity, we restrict ourselves only with a case of the unbroken PT-symmetry although nearly all results can be generalized to the case of spontaneous PT-symmetry breaking with pairs of eigenstates having mutually complex conjugated eigenvalues). The normalization (8) leads to the value $\gamma_{n}= \pm 1$. One can see that the biorthogonality does not, in general, provide positive binorms of states related by the PT-symmetry,

$$
\int_{-\infty}^{+\infty} \psi_{n}^{*}(-x) \psi_{m}(x) d x=\gamma_{n} \delta_{n m}= \pm \delta_{n m},
$$

bringing negative norm states in the PT-odd sector.

For non-Hermitian Hamiltonians one can formulate the extended eigenvalue problem, searching not only for normalizable eigenfunctions but also for normalizable associated functions for discrete part of the energy spectrum. Some related problems have been known for a long time in mathematics of linear differential equations (see for instance, 68]) .

Let us give the formal definition.

Definition 1. The function $\psi_{n, i}(x)$ is called a formal associated function of $i$-th order of the Hamiltonian $h$ for a spectral value $\lambda_{n}$, if

$$
\left(h-\lambda_{n}\right)^{i+1} \psi_{n, i} \equiv 0, \quad\left(h-\lambda_{n}\right)^{i} \psi_{n, i} \not \equiv 0,
$$

where 'formal' emphasizes that a related function is not necessarily normalizable.

In particular, the associated function of zero order $\psi_{n, 0}$ is a formal eigenfunction of $h$ (a solution of the homogeneous Schrödinger equation, not necessarily normalizable).

Let us single out normalizable associated functions and the case when $h$ maps them into normalizable functions 2 .

Evidently this may occur only for non-Hermitian Hamiltonians. Then for any normalizable associated functions $\psi_{n, i}(x)$ and $\psi_{n^{\prime}, i^{\prime}}(x)$ the transposition symmetry holds

$$
\int_{-\infty}^{+\infty} h \psi_{n, i}(x) \psi_{n^{\prime}, i^{\prime}}(x) d x=\int_{-\infty}^{+\infty} \psi_{n, i}(x) h \psi_{n^{\prime}, i^{\prime}}(x) d x .
$$

Furthermore one can prove the following relations:

$$
\int_{-\infty}^{+\infty} \psi_{n, i}(x) \psi_{n^{\prime}, i^{\prime}}(x) d x \equiv\left(\psi_{n, i}^{*}, \psi_{n^{\prime}, i^{\prime}}\right)=0, \quad \lambda_{n} \neq \lambda_{n^{\prime}}
$$

where $(\ldots, \ldots)$ is scalar product.

\footnotetext{
${ }^{2}$ It takes place for a certain class of potentials described in Sec. 6, see Part II of our paper.
} 
As well, let's take two normalizable associated functions $\psi_{n, k}(x)$ and $\psi_{n, k^{\prime}}(x)$ so that, in general, $k \neq k^{\prime}$ and there are two different sequences of associated functions for $i \leq k$ and $i^{\prime} \leq k^{\prime}$

$$
\psi_{n, i}(x)=\left(h-\lambda_{n}\right)^{k-i} \psi_{n, k}(x), \quad \psi_{n, i^{\prime}}(x)=\left(h-\lambda_{n}\right)^{k^{\prime}-i^{\prime}} \psi_{n, k^{\prime}}(x) .
$$

Then

$$
\int_{-\infty}^{+\infty} \psi_{n, i}(x) \psi_{n, i^{\prime}}(x) d x=\left(\psi_{n, i}^{*}, \psi_{n, i^{\prime}}\right)=0, \quad i+i^{\prime} \leq \max \left\{k, k^{\prime}\right\}-1 .
$$

In particular, for some normalizable associated function $\psi_{n, l}(x)$, the "self-orthogonality" [66] is realized,

$$
\int_{-\infty}^{+\infty} \psi_{n, l}^{2}(x) d x=0, \quad \psi_{n, l}(x)=(h-\lambda)^{i-l} \psi_{n, i}(x), \quad l=0, \ldots,\left[\frac{i-1}{2}\right] .
$$

All the above relations are derived from the symmetry of a Hamiltonian under transposition and the very definition of associate functions.

We proceed to the special class of Hamiltonians for which the spectrum is discrete and there is a complete biorthogonal system $\left\{\left|\psi_{n, a, i}\right\rangle,\left|\tilde{\psi}_{n, a, i}\right\rangle\right\}$ such that,

$$
\begin{aligned}
& h\left|\psi_{n, a, 0}\right\rangle=\lambda_{n}\left|\psi_{n, a, 0}\right\rangle, \quad\left(h-\lambda_{n}\right)\left|\psi_{n, a, i}\right\rangle=\left|\psi_{n, a, i-1}\right\rangle, \\
& h^{\dagger}\left|\tilde{\psi}_{n, a, p_{n, a}-1}\right\rangle=\lambda_{n}^{*}\left|\tilde{\psi}_{n, a, p_{n, a}-1}\right\rangle, \quad\left(h^{\dagger}-\lambda_{n}^{*}\right)\left|\tilde{\psi}_{n, a, p_{n, a}-i-1}\right\rangle=\left|\tilde{\psi}_{n, a, p_{n, a}-i}\right\rangle,
\end{aligned}
$$

where $n=0,1,2, \ldots$ is an index of an $h$ eigenvalue $\lambda_{n}$,

$a=1, \ldots, d_{n}$ is an index of a Jordan cell (block) for the given eigenvalue, $\lambda_{n}$;

$d_{n}$ is a number of Jordan cells for $\lambda_{n}$;

$i=0, \ldots, p_{n, a}-1$ is an index of associated function in the Jordan cell with indexes $n, a$ and $p_{n, a}$ is a dimension of this Jordan cell. We have taken a general framework which is applicable also for matrix and/or multidimensional Hamiltonians. But the main results of this and the next sections are guaranteed only for scalar one-dimensional Hamiltonians with local potentials.

We remark that the number $d_{n}$ is called a geometric multiplicity of the eigenvalue $\lambda_{n}$. For a scalar one-dimensional Schrödinger equation it cannot normally exceed 1 (but may reach 2 in specific cases of periodic potentials and of potentials unbounded from below). In turn, the sum $\sum_{a} p_{n, a}$ is called an algebraic multiplicity of the eigenvalue $\lambda_{n}$.

The completeness implies the biorthogonality relations

$$
\left\langle\tilde{\psi}_{n, a, i} \mid \psi_{m, b, j}\right\rangle=\delta_{n m} \delta_{a b} \delta_{i j}
$$

and the resolution of identity

$$
I=\sum_{n=0}^{+\infty} \sum_{a=1}^{d_{n}} \sum_{i=0}^{p_{n, a}-1}\left|\psi_{n, a, i}\right\rangle\left\langle\tilde{\psi}_{n, a, i}\right|
$$

The spectral decomposition for the Hamiltonian can be constructed as well,

$$
h=\sum_{n=0}^{+\infty} \sum_{a=1}^{d_{n}}\left[\lambda_{n} \sum_{i=0}^{p_{n, a}-1}\left|\psi_{n, a, i}\right\rangle\left\langle\tilde{\psi}_{n, a, i}\left|+\sum_{i=0}^{p_{n, a}-2}\right| \psi_{n, a, i}\right\rangle\left\langle\tilde{\psi}_{n, a, i+1}\right|\right] .
$$


It represents the analog of the block-diagonal Jordan form for arbitrary non-Hermitian matrices [69].

If existing such biorthogonal systems are not unique. Indeed the relations (19) remain invariant under the group of triangle transformations,

$$
\begin{aligned}
\left|\psi_{n, a, i}^{\prime}\right\rangle & =\sum_{0 \leq j \leq i} \alpha_{i j}\left|\psi_{n, a, j}\right\rangle, \\
\left|\tilde{\psi}_{n, a, k}^{\prime}\right\rangle & =\sum_{k \leq l \leq p_{n, a}-1} \beta_{k l}\left|\tilde{\psi}_{n, a, l}\right\rangle,
\end{aligned}
$$

where the matrix elements must obey the following equations,

$$
\begin{aligned}
& \alpha_{i j}=\alpha_{i+1, j+1}=\alpha_{i-j, 0} \equiv \alpha_{i-j}, \quad \alpha_{00} \neq 0, \\
& \beta_{k l}=\beta_{k+1, l+1}=\beta_{k-l+p_{n, a}-1 \equiv \beta_{k-l}, p_{n, a}-1}, \quad \beta_{p_{n, a}-1, p_{n, a}-1} \neq 0 .
\end{aligned}
$$

The biorthogonality (20) restricts the choice of pairs of matrices $\hat{\alpha}$ and $\hat{\beta}$ in (23) to be,

$$
\hat{\beta}^{\dagger}=\hat{\alpha}^{-1} \text {. }
$$

This freedom in the redefinition of the biorthogonal basis is similar to Eq. (8) and it can be exploited to define the pairs of biorthogonal functions $\psi_{n, a, i}(x) \equiv\left\langle x \mid \psi_{n, a, i}\right\rangle$ and $\tilde{\psi}_{n, a, i}(x) \equiv\left\langle x \mid \tilde{\psi}_{n, a, i}\right\rangle$ in accordance with (9). However one has to take into account our enumeration of associated functions $\psi_{n, a, i}(x)$ vs. their conjugated ones $\tilde{\psi}_{n, a, i}(x)$ as it is introduced in Eqs. (19)

$$
\psi_{n, a, i}(x)=\tilde{\psi}_{n, a, p_{n, a}-i-1}^{*}(x) \equiv\left\langle\tilde{\psi}_{n, a, p_{n, a}-i-1} \mid x\right\rangle .
$$

Then the analog of Eq. (9) reads,

$$
\int_{-\infty}^{+\infty} \psi_{n, a, i}(x) \psi_{m, b, p_{m, b}-j-1}(x) d x=\delta_{n m} \delta_{a b} \delta_{i j} .
$$

We stress that this kind of biorthogonal systems is determined uniquely up to an overall sign.

In these terms it becomes clear that the relations (17) have the meaning of orthogonality of some off-diagonal pairs in the biorthogonal system $\left\{\left|\psi_{n, a, i}\right\rangle,\left|\tilde{\psi}_{n, a, j}\right\rangle\right\}$ as

$$
\begin{gathered}
\psi_{n, a, i}(x)=\left(h-\lambda_{n}\right)^{p_{n, a}-1-i} \psi_{n, a, p_{n, a}-1}(x), \\
\tilde{\psi}_{n, a, j}^{*}(x)=\psi_{n, a, p_{n, a}-1-j}(x)=\left(h-\lambda_{n}\right)^{j} \psi_{n, a, p_{n, a}-1}(x) .
\end{gathered}
$$

When comparing with specification of indices in Eq. (17) one identifies

$p_{n, a}-1-j \leftrightarrow i, \quad i \leftrightarrow i^{\prime}$. In both cases $k=k^{\prime}=p_{n, a}-1$. Then the inequality (17) singles out off-diagonal binorms, $i \leq j-1$. From Eq. (28) it follows that in order to have all diagonal binorms non-vanishing it is sufficient to prove that at least one of them is not zero because

$$
\begin{aligned}
& \int_{-\infty}^{+\infty} \psi_{n, a, 0}(x) \psi_{n, a, p_{n, a}-1}(x) d x \\
& =\int_{-\infty}^{+\infty}\left[\left(h-\lambda_{n}\right)^{p_{n, a}-1} \psi_{n, a, p_{n, a}-1}(x)\right] \psi_{n, a, p_{n, a}-1}(x) d x \\
& =\int_{-\infty}^{+\infty} \psi_{n, a, i}(x) \psi_{n, a, p_{n, a}-1-i}(x) d x \neq 0 .
\end{aligned}
$$


The latter is necessary for the completeness of the basis because of the absence of biorthogonal pairs of basis elements made of bound and associated functions in the diagonal resolution of identity. If some of such pairs in resolution of identity (21) were biorthogonal then this operator would be at best a projector but not an identity.

For a scalar one-dimensional Schrödinger equation the geometric multiplicity $d_{n}$ of the eigenvalue $\lambda_{n}$ cannot normally exceed 1 . The latter possibility of non-degenerate eigenstates will be implied throughout this paper. Thereby in the rest of the paper the index $a=1=d_{n}$ will be omitted .

It is certainly interesting to examine what are specific features of biorthogonal bases for PT-symmetric systems. Let us restrict ourselves by one-dimensional systems with nondegenerate spectrum of eigenstates and with the unbroken PT-symmetry, $\lambda_{n}^{*}=\lambda_{n}$. First one can easily find that the functions $\tilde{\psi}_{n, p_{n}-i-1}(-x)$ are normalizable solutions of the initial Hamiltonian $h$ and therefore can be decomposed into a linear combination of its basis,

$$
\tilde{\psi}_{n, p_{n}-i-1}(-x)=\psi_{n, i}^{*}(-x)=\sum_{0 \leq j \leq i} \gamma_{n, i j} \psi_{n, j}(x),
$$

where $\gamma_{n, i j}=\gamma_{n, i-j}$ due to Eqs. conjugated to (19) . By complex conjugation of Eq.(30) and its further convolution with $\gamma_{n, l-i}$ (and after changing the sign $x \rightarrow-x$ ) one comes to the conditions on matrix elements,

$$
\left.\sum_{j \leq i \leq l} \gamma_{n, l-i} \gamma_{n, i-j}^{*}\right|_{j<l}=0 ; \quad \gamma_{n, 0}^{2}=1 .
$$

The analysis of the biorthogonality relations (20) and (27) for the PT-symmetric basis (30) leads to the conclusion that all numbers $\gamma_{n, i-j}$ are it real . Then one derives from (31) that $\left.\gamma_{n, i-j}\right|_{j<i}=0$ and the elements of a Jordan cell basis, the eigen- and associated functions are simultaneously PT-even or PT-odd depending on the sign of $\gamma_{n, 0}= \pm 1$,

$$
\tilde{\psi}_{n, p_{n}-i-1}(x)=\psi_{n, i}^{*}(x)=\gamma_{n, 0} \psi_{n, i}(-x) .
$$

Thus a biorthogonal basis of eigen-and associated functions exists which is fully compatible with PT-symmetry . As a consequence of (32) the integrals $\int_{-\infty}^{+\infty} \psi_{n, 0}(x) \psi_{n, p_{n}-1}^{*}(-x) d x$ remain real.

We remark that in general case the existence and the completeness of a biorthogonal system is not obvious (especially if the continuum spectrum is present [32]) and needs a careful examination.

\section{The origin of non-diagonalizable Hamiltonians is level confluence}

Let us demonstrate that one can generate a Jordan cell of a Hamiltonian in the process of coalescing levels of the Hamiltonian . In the simplest case one can consider a Hamiltonian depending on the parameter $\mu$,

$$
h_{\mu}=-\partial^{2}+V(x ; \mu)
$$

with two eigenfunctions $\psi_{1,2}(x ; \mu)$,

$$
h_{\mu} \psi_{1,2}(x ; \mu)=\lambda_{1,2}(\mu) \psi_{1,2}(x ; \mu) .
$$


Assume that the levels $\lambda_{1}(\mu)$ and $\lambda_{2}(\mu)$ coalesce for $\mu=\mu_{0}$ :

$$
\psi_{1}\left(x ; \mu_{0}\right) \equiv \psi_{2}\left(x ; \mu_{0}\right)=\varphi_{0}(x), \quad \lambda_{1}\left(\mu_{0}\right)=\lambda_{2}\left(\mu_{0}\right)=\lambda_{0} .
$$

Let us also suppose that the functions $\frac{\partial \psi_{1}}{\partial \mu}(x ; \mu)$ and $\frac{\partial \psi_{2}}{\partial \mu}(x ; \mu)$ are normalizable. Then, it is evident that

$$
\begin{gathered}
{\left[h_{\mu}-\lambda_{1,2}(\mu)\right] \frac{\partial \psi_{1,2}}{\partial \mu}=-\left[\frac{\partial V}{\partial \mu}-\lambda_{1,2}^{\prime}(\mu)\right] \psi_{1,2},} \\
\left(h_{\mu_{0}}-\lambda_{0}\right)\left[\frac{\partial \psi_{1}}{\partial \mu}\left(x ; \mu_{0}\right)-\frac{\partial \psi_{2}}{\partial \mu}\left(x ; \mu_{0}\right)\right]=\left[\lambda_{1}^{\prime}\left(\mu_{0}\right)-\lambda_{2}^{\prime}\left(\mu_{0}\right)\right] \varphi_{0}(x)
\end{gathered}
$$

and, thus, the functions

$$
\varphi_{0}(x), \quad \varphi_{1}(x)=\frac{\frac{\partial \psi_{1}}{\partial \mu}\left(x ; \mu_{0}\right)-\frac{\partial \psi_{2}}{\partial \mu}\left(x ; \mu_{0}\right)}{\lambda_{1}^{\prime}\left(\mu_{0}\right)-\lambda_{2}^{\prime}\left(\mu_{0}\right)}
$$

form a Jordan cell of the second order for the Hamiltonian $h_{0}=h_{\mu_{0}}$ :

$$
h_{0} \varphi_{0}=\lambda_{0} \varphi_{0}, \quad\left(h_{0}-\lambda_{0}\right) \varphi_{1}=\varphi_{0} .
$$

Let us proceed now to the case with three coalescing levels of $h_{\mu}$ :

$$
\begin{gathered}
{\left[h_{\mu}-\lambda_{j}(\mu)\right] \psi_{j}(x ; \mu)=0, \quad j=1,2,3,} \\
\psi_{1}\left(x ; \mu_{0}\right) \equiv \psi_{2}\left(x ; \mu_{0}\right) \equiv \psi_{3}\left(x ; \mu_{0}\right)=\varphi_{0}(x), \quad \lambda_{1}\left(\mu_{0}\right)=\lambda_{2}\left(\mu_{0}\right)=\lambda_{3}\left(\mu_{0}\right)=\lambda_{0} .
\end{gathered}
$$

Let us introduce the auxiliary functions

$$
\begin{gathered}
\psi_{1}^{(0)}(x ; \mu)=\left[1+\varkappa\left(\mu-\mu_{0}\right)\right] \psi_{1}(x ; \mu), \quad \psi_{j}^{(0)}(x ; \mu)=\psi_{j}(x ; \mu), \quad j=2,3, \\
\psi_{j}^{(1)}(x ; \mu)=\frac{\frac{\partial \psi_{j}^{(0)}}{\partial \mu}(x ; \mu)-\frac{\partial \psi_{3}^{(0)}}{\partial \mu}(x ; \mu)}{\lambda_{j}^{\prime}(\mu)-\lambda_{3}^{\prime}(\mu)}, \quad j=1,2,
\end{gathered}
$$

where the constant $\varkappa$ is chosen so that the associated functions of the first order

$$
\psi_{1}^{(1)}\left(x ; \mu_{0}\right)=\frac{\frac{\partial \psi_{1}}{\partial \mu}\left(x ; \mu_{0}\right)-\frac{\partial \psi_{3}}{\partial \mu}\left(x ; \mu_{0}\right)}{\lambda_{1}^{\prime}\left(\mu_{0}\right)-\lambda_{3}^{\prime}\left(\mu_{0}\right)}+\frac{\varkappa}{\lambda_{1}^{\prime}\left(\mu_{0}\right)-\lambda_{3}^{\prime}\left(\mu_{0}\right)} \varphi_{0}(x)
$$

and

$$
\psi_{2}^{(1)}\left(x ; \mu_{0}\right)=\frac{\frac{\partial \psi_{2}}{\partial \mu}\left(x ; \mu_{0}\right)-\frac{\partial \psi_{3}}{\partial \mu}\left(x ; \mu_{0}\right)}{\lambda_{2}^{\prime}\left(\mu_{0}\right)-\lambda_{3}^{\prime}\left(\mu_{0}\right)}
$$

(cf. with (33) $)$ are identica 3 . When using these auxiliary functions one can obtain the canonical set of associated functions,

$$
\varphi_{0}(x), \quad \varphi_{1}(x)=\psi_{1}^{(1)}\left(x ; \mu_{0}\right) \equiv \psi_{2}^{(1)}\left(x ; \mu_{0}\right), \quad \varphi_{2}(x)=\frac{\frac{\partial \psi_{1}^{(1)}}{\partial \mu}\left(x ; \mu_{0}\right)-\frac{\partial \psi_{2}^{(1)}}{\partial \mu}\left(x ; \mu_{0}\right)}{2\left[\lambda_{1}^{\prime}\left(\mu_{0}\right)-\lambda_{2}^{\prime}\left(\mu_{0}\right)\right]},
$$

which form Jordan cell of the third order for the Hamiltonian $h_{0}=h_{\mu_{0}}$ :

$$
h_{0} \varphi_{0}=\lambda_{0} \varphi_{0}, \quad\left(h_{0}-\lambda_{0}\right) \varphi_{j}=\varphi_{j-1}, \quad j=1,2 .
$$

\footnotetext{
${ }^{3}$ The constant $\varkappa$ exists because in one-dimensional case the difference of normalizable associated functions of the first order for the same eigenfunction is proportional to this eigenfunction. This freedom has been discussed in the previous section, see Eq.(23) .
} 
Thus, we have shown that the confluence of two (three) levels (of algebraic multiplicity 1) leads to appearance of a Jordan cell of the second (third) order. The described construction is illustrated by an example in Subsec. 7.2. In the case of confluence of a larger number $n$ of levels (of algebraic multiplicity 1) one can construct the canonical chain of $n$ normalizable eigenfunction and associated functions in the same way.

One can examine also the confluence of levels of different algebraic multiplicity. Let us restrict ourselves to the simplest case, when for the Hamiltonian $h_{\mu}$ level $\lambda_{1}(\mu)$ of algebraic multiplicity 2 coalesces for $\mu=\mu_{0}$ with level $\lambda_{2}(\mu)$ of algebraic multiplicity 1 :

$$
\begin{gathered}
h_{\mu} \psi_{10}=\lambda_{1}(\mu) \psi_{10}, \quad\left[h_{\mu}-\lambda_{1}(\mu)\right] \psi_{11}=\psi_{10}, \\
h_{\mu} \psi_{2}=\lambda_{2}(\mu) \psi_{2}, \\
\psi_{10}\left(x ; \mu_{0}\right) \equiv \psi_{2}\left(x ; \mu_{0}\right)=\varphi_{0}(x), \quad \lambda_{1}\left(\mu_{0}\right)=\lambda_{2}\left(\mu_{0}\right)=\lambda_{0} .
\end{gathered}
$$

Again we introduce the auxiliary functions

$$
\begin{aligned}
\psi_{1}^{(0)}(x ; \mu) & =\psi_{10}(x ; \mu), \quad \psi_{2}^{(0)}(x ; \mu)=\left[1+\varkappa\left(\mu-\mu_{0}\right)\right] \psi_{2}(x ; \mu), \\
\psi_{1}^{(1)}(x ; \mu) & =\psi_{11}(x ; \mu), \quad \psi_{2}^{(1)}(x ; \mu)=\frac{\frac{\partial \psi_{1}^{(0)}}{\partial \mu}(x ; \mu)-\frac{\partial \psi_{2}^{(0)}}{\partial \mu}(x ; \mu)}{\lambda_{1}^{\prime}(\mu)-\lambda_{2}^{\prime}(\mu)},
\end{aligned}
$$

where one should choose $\varkappa$ so that the associated functions of the first order $\psi_{1}^{(1)}\left(x ; \mu_{0}\right) \equiv \psi_{11}\left(x ; \mu_{0}\right)$ and

$$
\psi_{2}^{(1)}\left(x ; \mu_{0}\right) \equiv \frac{\frac{\partial \psi_{10}}{\partial \mu}\left(x ; \mu_{0}\right)-\frac{\partial \psi_{2}}{\partial \mu}\left(x ; \mu_{0}\right)}{\lambda_{1}^{\prime}\left(\mu_{0}\right)-\lambda_{2}^{\prime}\left(\mu_{0}\right)}-\frac{\varkappa}{\lambda_{1}^{\prime}\left(\mu_{0}\right)-\lambda_{2}^{\prime}\left(\mu_{0}\right)} \varphi_{0}(x),
$$

are identical. Therefrom one can get the appropriate set of associated functions

$$
\begin{aligned}
& \varphi_{0}(x), \quad \varphi_{1}(x)=\psi_{1}^{(1)}\left(x ; \mu_{0}\right) \equiv \psi_{2}^{(1)}\left(x ; \mu_{0}\right), \\
& \varphi_{2}(x)=\frac{\frac{\partial \psi_{2}^{(1)}}{\partial \mu}\left(x ; \mu_{0}\right)-2 \frac{\partial \psi_{1}^{(1)}}{\partial \mu}\left(x ; \mu_{0}\right)}{2\left[\lambda_{2}^{\prime}\left(\mu_{0}\right)-\lambda_{1}^{\prime}\left(\mu_{0}\right)\right]}
\end{aligned}
$$

which form Jordan cell of the third order for the Hamiltonian $h_{0}=h_{\mu_{0}}$ :

$$
h_{0} \varphi_{0}=\lambda_{0} \varphi_{0}, \quad\left(h_{0}-\lambda_{0}\right) \varphi_{j}=\varphi_{j-1}, \quad j=1,2 .
$$

\section{Non-linear SUSY for complex potentials}

Supersymmetric Quantum Mechanics (SUSY QM) in one dimension represents a concise way for an almost isospectral transformation between two quantum systems [70] - 78] (see the reviews [79] - [84] ). Conventionally it can be built for a pair of Hamiltonians $h^{+}$ and $h^{-}$assembled into a Super-Hamiltonian,

$$
H=\left(\begin{array}{cc}
h^{+} & 0 \\
0 & h^{-}
\end{array}\right)=\left(\begin{array}{cc}
-\partial^{2}+V_{1}(x) & 0 \\
0 & -\partial^{2}+V_{2}(x)
\end{array}\right) \equiv-\partial^{2} \mathbf{I}+\mathbf{V}(x),
$$

where the potential $\mathbf{V}(x)$ is, in general, complex. The (almost) isospectral connection between $h^{+}$and $h^{-}$is realized by the intertwining relations ,

$$
h^{+} q^{+}=q^{+} h^{-}, \quad q^{-} h^{+}=h^{-} q^{-},
$$


with $q^{ \pm}$being components of the supercharges,

$$
Q=\left(\begin{array}{cc}
0 & q^{+} \\
0 & 0
\end{array}\right), \quad \bar{Q}=\left(\begin{array}{cc}
0 & 0 \\
q^{-} & 0
\end{array}\right), \quad Q^{2}=\bar{Q}^{2}=0 .
$$

The isospectral relations (36) result in the conservation of supercharges or the supersymmetry of the Super-Hamiltonian,

$$
[H, Q]=[H, \bar{Q}]=0,
$$

In general, its algebraic closure is given, by a non-linear (deformed) SUSY algebra,

$$
\{Q, \bar{Q}\}=\mathcal{P}(H)
$$

where $\mathcal{P}(H)$ is a function of the Super-Hamiltonian [34, 83, 85].

The relevant supercharges are supposed to be generated by $N$-th order differential operators with smooth coefficient functions $w_{k}^{ \pm}(x)$ :

$$
q^{ \pm} \equiv q_{N}^{ \pm}=\sum_{k=0}^{N} w_{k}^{ \pm}(x) \partial^{k}, \quad w_{N}^{ \pm}=\text {const } \equiv(\mp 1)^{N} .
$$

We focus our analysis on the non-Hermitian Hamiltonians interrelated by complex supercharges, i.e. the supercharges with complex and smooth coefficient functions $w_{k}^{ \pm}(x)$. In this paper we choose $q_{N}^{-}$connected to $q_{N}^{+}$by means of ${ }^{t}$ - transposition, $q_{N}^{-}=\left(q_{N}^{+}\right)^{t}$. Evidently the Super-Hamiltonian of Schrödinger type is self-transposed ( ${ }^{t}$-symmetric) as only the scalar potentials are under consideration.

The algebraic structure of a Non-linear SUSY for local Hamiltonians is exhaustively determined by the following theorem,

Theorem 1: on SUSY algebras with transposition symmetry

Let us introduce two sets of $N$ linearly independent functions

$\phi_{n}^{ \pm}(x)(n=1, \cdots N)$ which represent complete sets of zero-modes of the supercharge components,

$$
q_{N}^{ \pm} \phi_{n}^{ \pm}=0, \quad q_{N}^{-}=\left(q_{N}^{+}\right)^{t}
$$

Then:

1) the Hamiltonians $h^{ \pm}$have finite matrix representations when acting on the set of functions $\phi_{n}^{ \pm}(x)$,

$$
h^{ \pm} \phi_{n}^{\mp}=\sum_{m} S_{n m}^{ \pm} \phi_{m}^{\mp}
$$

2) the SUSY algebra closure with $\bar{Q}=Q^{t}$ takes the polynomial form,

$$
\left\{Q, Q^{t}\right\}=\operatorname{det}\left[E \mathbf{I}-\mathbf{S}^{+}\right]_{E=H}=\operatorname{det}\left[E \mathbf{I}-\mathbf{S}^{-}\right]_{E=H} \equiv \mathcal{P}_{N}(H) .
$$

The proof in $\left[85\right.$ is based on the quasi-diagonalization of matrices $\mathbf{S}^{ \pm}$, i.e. on their reduction to the Jordan canonical form $\widetilde{\mathbf{S}}^{ \pm}$which is block-diagonal. Such a diagonalization 
can be realized by non-degenerate linear transformations $\Omega^{ \pm}$of the zero-mode sets which induce the similarity transformations of matrices $\mathbf{S}^{ \pm}$,

$$
\begin{aligned}
\tilde{\phi}_{l}^{ \pm} & =\sum_{m=1}^{N} \Omega_{l m}^{\mp} \phi_{m}^{ \pm}, \quad h^{ \pm} \tilde{\phi}_{l}^{\mp}=\sum_{m=1}^{N} \widetilde{S}_{l m}^{ \pm} \tilde{\phi}_{m}^{\mp}, \\
\widetilde{\mathbf{S}}^{ \pm} & =\Omega^{ \pm} \mathbf{S}^{ \pm}\left(\Omega^{ \pm}\right)^{-1} .
\end{aligned}
$$

Evidently the canonical bases of zero-modes of intertwining operators $q_{N}^{ \pm}$form the set of (in general, formal - not necessarily normalizable) solutions and associated functions of the Hamiltonians $h^{\mp}$. These elements of canonical bases of intertwining operator kernels are named as transformation functions. The proof in [85] can be easily generalized to the complex case with transposition symmetry being built up along the same scheme.

For the polynomial SUSY algebra there is a possibility that the intertwining operators may be trivially reduced by a factor depending on the Hamiltonian, without any changes in the Hamiltonians $h^{ \pm}$, namely,

$$
q_{N}^{ \pm}=P\left(h^{ \pm}\right) p_{M}^{ \pm}=p_{M}^{ \pm} P\left(h^{\mp}\right),
$$

where $P(x)$ is assumed to be a polynomial and $N \geq M+2$. Thus some of the roots of associated polynomials may not be involved in determination of the structure of the potentials.

This problem of disentangling the nontrivial part of a supercharge and avoiding multiple SUSY algebras generated by means of "dressing" can be systematically tackled with the help of the following theorem which can be also extended for complex potentials.

\section{"Strip-off" Theorem 2 .}

Let's assume the construction of the Theorem on SUSY algebras with transposition symmetry. Then the requirement

that the matrix $\widetilde{\mathbf{S}}^{-}$(or $\widetilde{\mathbf{S}}^{+}$) generated on the subspace of zero-modes of the operator $q_{N}^{+}$ (or $q_{N}^{-}$) contains $m$ pairs (and no more) of Jordan cells with equal eigenvalues $\lambda_{l}$ in each pair and the sizes $\delta k_{l}$ and $k_{l}+\delta k_{l}$ ( $\delta k_{l}$ being the size of a smallest cell in the $l$-th pair) is necessary and sufficient to ensure that the intertwining operator $q_{N}^{+}$(or $q_{N}^{-}$) can be factorized:

$$
q_{N}^{ \pm}=p_{M}^{ \pm} \prod_{l=1}^{m}\left(\lambda_{l}-h^{\mp}\right)^{\delta k_{l}},
$$

where $p_{M}^{ \pm}$are intertwining operators of order $M=N-2 \sum_{l=1}^{m} \delta k_{l}=\sum_{j=1}^{n} k_{j}$ which cannot be decomposed further on in the product (46) type. Herein $k_{j}$ for $m+1 \leq j \leq n$ are sizes of unpaired Jordan cells .

Remark 1. The matrices $\widetilde{\mathbf{S}}^{ \pm}$cannot contain more than two Jordan cells with the same eigenvalue $\lambda$ because otherwise the operator $\lambda-h^{ \pm}$would have more than two linearly independent zero-mode solutions.

Remark 2. This theorem together with the Theorem 1 entails the essential identity of the Jordan forms $\widetilde{\mathbf{S}}^{-}$and $\widetilde{\mathbf{S}}^{+}$(up to transposition of certain Jordan cells).

Remark 3. The supercharge components cannot be stripped-off if the polynomial $\mathcal{P}_{N}(x)$ does not have degenerate zeroes. The latter is sufficient to deal with SUSY charges nontrivially factorizable, but not necessary because degenerate zeroes may well arise in the 
ladder (dressing chain) construction giving new pairs of isospectral potentials.

Remark 4. In general, a Super-Hamiltonian may commute with several different supercharges. In this case few hidden-symmetry operators arise. The optimization of such a system of supercharges till one or two independent ones, their stripping-off and the minimal structure of a symmetry operator has been investigated in [85] in details for Hermitian Hamiltonians. In so far as in the quoted paper the transposition was used as a main conjugation operation, all essential results of [85] remain to be valid also for complex potentials.

The intertwining operators (supercharge components) can be formally factorized into the products of elementary Darboux operators. Let $\phi_{j}^{-} \equiv \phi_{j}, j=1, \ldots, N$ be 4 the basis, in which the $\mathbf{S}^{+}$-matrix (see Th. 1) has a canonical Jordan form and $\lambda_{j}$ is an eigenvalue of $\mathbf{S}^{+}$corresponding to the Jordan cell, to which $\phi_{j}$ belongs. Then adapting the Lemma 1 from 85] for non-Hermitian Hamiltonians one can prove the following statements:

1) for the supercharge components the factorization holds, in particular,

$$
q_{N}^{-}=r_{N}^{-} \ldots r_{1}^{-}
$$

where the Darboux operators

$$
r_{j}^{-}=\partial+\chi_{j}(x), \quad j=1, \ldots, N,
$$

can be chosen so that

$$
r_{j}^{-} \ldots r_{1}^{-} \phi_{N-j+1}=0, \quad j=1, \ldots, N
$$

2) the chain relations take place

$$
\begin{gathered}
\left(r_{l+1}^{-}\right)^{t} r_{l+1}^{-}+\lambda_{l+1}=r_{l}^{-}\left(r_{l}^{-}\right)^{t}+\lambda_{l} \equiv h_{l}, \quad j=1, \ldots, N-1, \\
\left(r_{1}^{-}\right)^{t} r_{1}^{-}+\lambda_{1}=h^{+} \equiv h_{0}, \quad r_{N}^{-}\left(r_{N}^{-}\right)^{t}+\lambda_{N}=h^{-} \equiv h_{N} ;
\end{gathered}
$$

3) the intermediate Hamiltonians $h_{l}, l=1, \ldots, N-1$ have Schrödinger form:

$$
\begin{gathered}
h_{l}=-\partial^{2}+v_{l}(x), \quad v_{l}(x)=\chi_{l+1}^{2}(x)-\chi_{l+1}^{\prime}(x)+\lambda_{l+1}=\chi_{l}^{2}(x)+\chi_{l}^{\prime}(x)+\lambda_{l}, \\
V_{1}(x) \equiv v_{0}(x)=\chi_{1}^{2}(x)-\chi_{1}^{\prime}(x)+\lambda_{1}, \quad V_{2}(x) \equiv v_{N}(x)=\chi_{N}^{2}(x)+\chi_{N}^{\prime}(x)+\lambda_{N},
\end{gathered}
$$

but, in general, with complex and/or singular potentials;

4) the intertwining relations are valid:

$$
h_{l}\left(r_{l+1}^{-}\right)^{t}=\left(r_{l+1}^{-}\right)^{t} h_{l+1}, \quad r_{l+1}^{-} h_{l}=h_{l+1} r_{l+1}^{-}, \quad l=0, \ldots, N-1 .
$$

Let us introduce the generalized Crum determinants made of solutions of the initial Schrödinger equation for the Hamiltonian $h^{+}$as well as of some of its formal associated functions,

$$
w_{j}(x)=\left|\begin{array}{cccc}
\phi_{N}(x) & \phi_{N}^{\prime}(x) & \ldots & \phi_{N-1)}^{(j-1)}(x) \\
\phi_{N-1}(x) & \phi_{N-1}^{\prime}(x) & \ldots & \phi_{N-1}^{(j-1)}(x) \\
\ldots \ldots \ldots \ldots & \ldots \ldots \ldots \ldots \ldots \ldots & \ldots \ldots \ldots \\
\phi_{N-j+1}(x) & \phi_{N-j+1}^{\prime}(x) & \ldots & \phi_{N-j+1}^{(j-1)}(x)
\end{array}\right|, \quad j=1, \ldots, N .
$$

\footnotetext{
${ }^{4}$ In what follows, in the notations for $\phi_{j}^{\mp}$, we omit their relation to a Hamiltonian $h^{ \pm}$when it is only one of these sets which is used, in order to avoid too heavy indices.
} 
Then in virtue of Eq. (48) one finds the representation for the intertwining operators,

$$
r_{j}^{-} \ldots r_{1}^{-}=\frac{1}{w_{j}(x)}\left|\begin{array}{cccc}
\phi_{N}(x) & \phi_{N}^{\prime}(x) & \ldots & \phi_{N}^{(j)}(x) \\
\ldots \ldots \ldots \ldots \ldots \ldots \ldots \ldots \ldots \ldots & \ldots & \ldots \ldots \ldots \ldots \\
\phi_{N-j+1}(x) & \phi_{N-j+1}^{\prime}(x) & \ldots & \phi_{N-j+1}^{(j-1)}(x) \\
1 & \partial & \ldots & \partial^{j}
\end{array}\right|, \quad j=1, \ldots, N
$$

and, consequently,

$$
r_{j}^{-} \ldots r_{1}^{-} \phi_{N-j}=\frac{w_{j+1}(x)}{w_{j}(x)}, \quad j=1, \ldots, N-1 .
$$

Hence the intermediate superpotentials are uniquely determined by the chosen basis of solutions and formal associated functions of a given Hamiltonian $h^{+}$for a given ordering

$$
\chi_{j}(x)=-\frac{\left[w_{j}(x) / w_{j-1}(x)\right]^{\prime}}{w_{j}(x) / w_{j-1}(x)}=-\frac{w_{j}^{\prime}(x)}{w_{j}(x)}+\frac{w_{j-1}^{\prime}(x)}{w_{j-1}(x)}, \quad j=1, \ldots, N, \quad w_{0}(x) \equiv 1 .
$$

Thus, from Eq. (50) one obtains the chain relations between intermediate potentials,

$$
v_{j}(x)-v_{j-1}(x)=2 \chi_{j}^{\prime}(x)=-2\left[\ln \frac{w_{j}(x)}{w_{j-1}(x)}\right]^{\prime \prime}, \quad j=1, \ldots, N
$$

and furthermore

$$
v_{j}(x)-v_{0}(x)=-2\left[\ln w_{j}(x)\right]^{\prime \prime}, \quad j=0, \ldots, N .
$$

It leads finally to the connection between the components of the potential in the SuperHamiltonian,

$$
V_{2}(x)=V_{1}(x)-2\left[\ln w_{N}(x)\right]^{\prime \prime} .
$$

The above set of relations is well-known from the Crum theory [86]. However here we have extended them including not only solutions of a Schrödinger equation but also its formal associated functions.

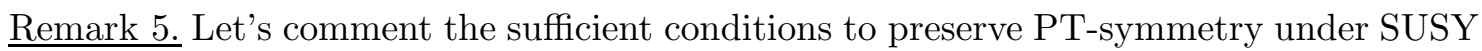
transformations, i.e. after intertwining with a Darboux-Crum operator . They consist in requirement for all transformation functions (all elements of the zero-mode basis of the intertwining operator) to be symmetric or antisymmetric in respect to PT-reflection . Indeed the PT-(anti)symmetry of basis elements entails the PT-(anti)symmetry of their Wronskian wherefrom one derives the PT-antisymmetry of $W^{\prime} / W$ and the PT-symmetry of $V_{2}=V_{1}-2(\ln W)^{\prime \prime}$. This is why under such conditions the above presented theorems and the forthcoming ones are certainly valid .

\section{Non-diagonalizable Hamiltonians and normal- izability of associated functions}

In this section we start examination of how the Darboux transformation may change the structure of a non-diagonalizable Hamiltonian with a Jordan cell spanned by a set of associated functions. We keep in mind that an intertwining operator may annihilate part 
of them as zero modes. As a result we find the additive composition of Jordan cells for partner Hamiltonians $h^{ \pm}$mediated by a Jordan cell for the Hamiltonian mapping of the zero-mode subspace of the intertwining operator $q_{N}^{-}$.

The rigorous results can be obtained with a specification of the class of potentials invariant under Darboux transformations. For such a class the asymptotic normalizability of associated functions at one of the infinities is preserved by Darboux transformations and will be described in certain lemmas and corollaries. When the normalizability on the whole axis is achieved we call the related associated functions as normalizable in general. The detailed mathematical proofs are given in part II, here we give only the general ideas of the construction as well as the formulations of the theorems and lemmas and the related corollaries .

Let us investigate the Jordan structure of the Hamiltonians $h^{ \pm}$. In what follows we restrict ourselves to the particular class of potentials:

Definition 2. Let $K$ be the set of all potentials $V(x)$ such that:

1) $V(x) \in C_{\mathbb{R}}^{\infty}$

2) there are $R_{0}>0$ and $\varepsilon>0\left(R_{0}\right.$ and $\varepsilon$ depend on $\left.V(x)\right)$ such that for any $|x| \geq R_{0}$ the inequality $\operatorname{Re} V(x) \geq \varepsilon$ takes place;

3) $\operatorname{Im} V(x) / \operatorname{Re} V(x)=o(1), x \rightarrow \pm \infty$ (this is sufficient to ensure the reality of the continuous spectrum);

4) functions

$$
\left(\int_{ \pm R_{0}}^{x} \sqrt{\left|V\left(x_{1}\right)\right|} d x_{1}\right)^{2}\left(\frac{\left|V^{\prime}(x)\right|^{2}}{|V(x)|^{3}}+\frac{\left|V^{\prime \prime}(x)\right|}{|V(x)|^{2}}\right)
$$

are bounded respectively for $x \geq R_{0}$ and $x \leq-R_{0}$.

$\underline{\text { Remark } 6 .}$ The last condition is not very rigid: it is fulfilled (if $x \rightarrow+\infty$ ), for example, for potentials:

1)

$$
\begin{gathered}
V(x)=a x^{\gamma}[1+o(1)], \quad V^{\prime}(x)=a \gamma x^{\gamma-1}[1+o(1)] \\
V^{\prime \prime}(x)=a \gamma(\gamma-1) x^{\gamma-2}+o\left(x^{\gamma-2}\right), \quad a>0, \quad \gamma>0 ; \\
V(x)=V_{0}+a x^{-\gamma}[1+o(1)], \quad V^{\prime}(x)=-a \gamma x^{-\gamma-1}[1+o(1)], \\
V^{\prime \prime}(x)=a \gamma(\gamma+1) x^{-\gamma-2}[1+o(1)], \quad V_{0}>0, \quad a \in \mathbb{C}, \quad \operatorname{Re} \gamma>0 ; \\
V(x)=a x^{\alpha} e^{b x^{\beta}}[1+o(1)], \quad V^{\prime}(x)=a b \beta x^{\alpha+\beta-1} e^{b x^{\beta}}[1+o(1)], \\
V^{\prime \prime}(x)=a b^{2} \beta^{2} x^{\alpha+2 \beta-2} e^{b x^{\beta}}[1+o(1)], \quad a>0, \quad b>0, \quad \alpha \in \mathbb{R}, \quad \beta>0 ; \\
V(x)=V_{0}+a x^{\alpha} e^{-b x^{\beta}}[1+o(1)], \quad V^{\prime}(x)=-a b \beta x^{\alpha+\beta-1} e^{-b x^{\beta}}[1+o(1)], \\
V^{\prime \prime}(x)=a b^{2} \beta^{2} x^{\alpha+2 \beta-2} e^{-b x^{\beta}}[1+o(1)], \quad V_{0}>0, a \in \mathbb{C}, \operatorname{Re} b>0, \alpha \in \mathbb{C}, \beta>0 .
\end{gathered}
$$

A similar statement is valid for $x \rightarrow-\infty$. Thus one can find in this class of potentials both representatives with purely discrete spectrum and the Hamiltonians with continuum spectrum .

Remark7. This class of potentials only partially overlaps with PT-symmetric set of potentials investigated recently [9]-[17], 23], 24] . We call the related class of Hamiltonians 
as softly non-Hermitian as they certainly don't involve complex coordinates in definition of asymptotic boundary conditions.

The set $K$ is closed under intertwining of Hamiltonians, that follows from the

Lemma 1: on invariance of the potential set $K$. Let: 1) $h^{+}=-\partial^{2}+V_{1}(x), V_{1}(x) \epsilon$ $K$; 2) $h^{-}=-\partial^{2}+V_{2}(x), V_{2}(x) \in C_{\mathbb{R}}$; 3) $q_{N}^{-} h^{+}=h^{-} q_{N}^{-}$, where $q_{N}^{-}$is differential operator of $N$ th order with coefficients from $C_{\mathbb{R}}^{2} ; 4$ ) each eigenvalue of $\mathbf{S}^{+}$-matrix of $q_{N}^{-}$(see Th. 1) satisfies one of the conditions: either $\lambda \leq 0$ or $\operatorname{Im} \lambda \neq 0$. Then: 1) $V_{2}(x) \in K$; 2) coefficients of $q_{N}^{-}$belong to $C_{\mathbb{R}}^{\infty}$; 3) $h^{+} q_{N}^{+}=q_{N}^{+} h^{-}$, where $q_{N}^{+}=\left(q_{N}^{-}\right)^{t}$, and moreover coefficients of $q_{N}^{+}$belong to $C_{\mathbb{R}}^{\infty}$ also.

Let us now analyze the normalizability properties of associated functions.

Definition 3. A function $f(x)$ is called normalizable at $+\infty$ (at $-\infty)$, if there is $R_{+}$ $\left(R_{-}\right)$such that

$$
\int_{R_{+}}^{+\infty}|f(x)|^{2} d x<+\infty \quad\left(\int_{-\infty}^{R_{-}}|f(x)|^{2} d x<+\infty\right) .
$$

Otherwise $f(x)$ is called non-normalizable at $+\infty$ (at $-\infty)$.

Using the asymptotics of formal associated functions (see lemma 9, part II), one can show that:

1) when the potential in $h$ (by which, in what follows, we imply either $h^{+}$or $h^{-}$) belongs to the class $K$, one can show that any formal associated function of $n$-th order of $h$, normalizable at $+\infty$ or $-\infty$ respectively, for the spectral value $\lambda$, satisfying either $\lambda \leq 0$ or $\operatorname{Im} \lambda \neq 0$, can be decomposed as follows

$$
\sum_{j=0}^{n} a_{j, \uparrow \downarrow} \varphi_{j, \uparrow \downarrow}(x), \quad a_{j, \uparrow \downarrow}=\text { Const }, \quad a_{n, \uparrow \downarrow} \neq 0,
$$

where $\varphi_{j, \uparrow \downarrow}(x), j \geq 0$ stand for either $\varphi_{j, \uparrow}(x)$ or $\varphi_{j, \downarrow}(x)$ and they form a sequence of associated functions normalizable at $+\infty$ or $-\infty$ respectively,

$$
h \varphi_{0, \uparrow \downarrow}=\lambda \varphi_{0, \uparrow \downarrow}, \quad(h-\lambda) \varphi_{j, \uparrow \downarrow}=\varphi_{j-1, \uparrow \downarrow}, \quad j \geq 1
$$

Correspondingly, any associated function of $n$-th order of $h$, non-normalizable at the same $+\infty$ or $-\infty$, for the same spectral value $\lambda$ can be presented as follows

$$
\sum_{j=0}^{n}\left(b_{j, \uparrow \downarrow} \varphi_{j, \uparrow \downarrow}(x)+c_{j, \uparrow \downarrow} \hat{\varphi}_{j, \uparrow \downarrow}(x)\right),
$$

where $b_{j, \uparrow \downarrow}, c_{j, \uparrow \downarrow}=$ const, either $b_{n, \uparrow \downarrow} \neq 0$ or $c_{n, \uparrow \downarrow} \neq 0$ and $\hat{\varphi}_{j, \uparrow \downarrow}(x), j \geq 0$ form a sequence of non-normalizable at $+\infty$ or $-\infty$ respectively associated functions

$$
h \hat{\varphi}_{0, \uparrow \downarrow}=\lambda \hat{\varphi}_{0, \uparrow \downarrow}, \quad(h-\lambda) \hat{\varphi}_{j, \uparrow \downarrow}=\hat{\varphi}_{j-1, \uparrow \downarrow}, \quad j \geq 1
$$

2) for the Hamiltonian with a potential from the class $K$, there are no degenerate eigenvalues, satisfying either $\lambda \leq 0$ or $\operatorname{Im} \lambda \neq 0$, i.e. the eigenvalues, whose geometric 
multiplicity exceeds 1 (the eigenvalues, for which there are more than one linearly independent eigenfunctions). Hence, for the Hamiltonian with a potential from $K$ there is no more than one Jordan cell made of an eigenfunction and associated functions, normalizable on the whole axis, for any given eigenvalue $\lambda$ such that either $\lambda \leq 0$ or $\operatorname{Im} \lambda \neq 0$.

Properties of associated functions under intertwining are described by the

Lemma 2. Let: 1) the conditions of the lemma 1 take place; 2) $\varphi_{n}(x), n=0, \ldots M$ be a sequence of associated functions of $h^{+}$for the spectral value $\lambda$ :

$$
h^{+} \varphi_{0}=\lambda \varphi_{0}, \quad\left(h^{+}-\lambda\right) \varphi_{n}=\varphi_{n-1}, \quad n \geq 1,
$$

where either $\lambda \leq 0$ or $\operatorname{Im} \lambda \neq 0$. Then:

1) there is a number $m$ such that $0 \leq m \leq \min \{M+1, N\}$,

$$
q_{N}^{-} \varphi_{n} \equiv 0, \quad n<m
$$

and

$$
\psi_{l}=q_{N}^{-} \varphi_{m+l}, \quad l=0, \ldots, M-m
$$

is a sequence of associated functions of $h^{-}$for the spectral value $\lambda$ :

$$
h^{-} \psi_{0}=\lambda \psi_{0}, \quad\left(h^{-}-\lambda\right) \psi_{l}=\psi_{l-1}, \quad l \geq 1 ;
$$

2) if the function $\varphi_{n}(x)$, for a given $0 \leq n \leq M$, is normalizable at $+\infty$ (on $\left.-\infty\right)$, then the function $q_{N}^{-} \varphi_{n}$ is normalizable at $+\infty($ on $-\infty)$ as well.

Corollary 1. Since $h^{+}$is an intertwining operator for itself and both eigenvalues of its $\mathbf{S}^{+}$-matrix are zero, then if $\varphi_{n}(x)$ is normalizable at $+\infty($ at $-\infty)$, then $\varphi_{j}(x), j=0$, $\ldots n-1$ are normalizable at $+\infty($ at $-\infty)$ as well.

Corollary 2. If there is an associated normalizable function $\varphi_{n}(x)$ of $n$-th order of the Hamiltonian $h$ with a potential from $K$, for an eigenvalue $\lambda$, which is either $\lambda \leq 0$ or $\operatorname{Im} \lambda \neq 0$, then for this eigenvalue there is an associated function $\varphi_{j}(x)$ of Hamiltonian $h$, normalizable on the whole axis, of any smaller order $j$ :

$$
\varphi_{j}=(h-\lambda)^{n-j} \varphi_{n}, \quad j=0, \ldots, n-1 .
$$

Corollary 3. Let $\varphi_{i, j}^{-}(x)$ be a canonical basis of zero-modes of the intertwining operator $q_{N}^{-}$, i.e. such that $\mathbf{S}^{+}$-matrix (from the Theorems 1 and 2 ) has in this basis the canonical (Jordan) form:

$$
h^{+} \varphi_{i, 0}^{-}=\lambda_{i} \varphi_{i, 0}^{-}, \quad\left(h^{+}-\lambda_{i}\right) \varphi_{i, j}^{-}=\varphi_{i, j-1}^{-}, \quad j=1, \ldots, k_{i}-1,
$$

where $k_{i}$ is a rank of a Jordan cell for $\lambda_{i}$. Then there are numbers $k_{i \uparrow}^{+}$and $k_{i \downarrow}^{+}, 0 \leq k_{i \uparrow, \downarrow}^{+} \leq k_{i}$ related to the Hamiltonian $h^{+}$such that for any $i$ the functions

$$
\varphi_{i, j}^{-}(x), \quad j=0, \ldots, k_{i \uparrow, \downarrow}^{+}-1
$$

are normalizable at $+\infty$ or $-\infty$ respectively and the functions

$$
\varphi_{i, j}^{-}(x), \quad j=k_{i \uparrow, \downarrow}^{+}, \ldots, k_{i}-1
$$


are non-normalizable at the same $+\infty$ or $-\infty$. Thus one can derive that the number of functions $\varphi_{i, j}^{-}(x)$ normalizable on the whole axis is equivalent to $\min \left\{k_{i \uparrow}^{+}, k_{i \downarrow}^{+}\right\}$and the number of functions $\varphi_{i, j}^{-}(x)$ non-normalizable at both ends is given by $k_{i}-\max \left\{k_{i \uparrow}^{+}, k_{i \downarrow}^{+}\right\}$.

Independence of these numbers $k_{i \uparrow, \downarrow}^{+}$on a choice of the canonical basis, in the case of stripped-off $q_{N}^{-}$, follows from

Lemma 3. Let: 1) conditions of lemma 1 take place; 2) $q_{N}^{-}$may not be stripped-off. Then any two formal associated functions of $h^{+}$of the same order for the same spectral value $\lambda$, when being zero-modes of $q_{N}^{-}$, are either simultaneously normalizable at $+\infty$ or simultaneously non-normalizable at $+\infty$. The same takes place at $-\infty$.

We refer the reader for the proofs of the Lemmas $1-3$ to the forthcoming Part II.

\section{Interrelation between Jordan cells in SUSY part- ners}

The first result on interrelation between Jordan structures of intertwined Hamiltonians and on the behavior of transformation functions at $\pm \infty$ follows from the

Lemma 4. Let us assume that: 1) conditions of lemma 1 take place; 2) $\left\{\varphi_{i, j}^{-}\right\}$and $\left\{\varphi_{i, j}^{+}\right\}$are canonical bases of $\operatorname{ker} q_{N}^{-}$and $\operatorname{ker} q_{N}^{+}$respectively; 3) $q_{N}^{-}$cannot be stripped-off; 4) $k_{i}$ is an algebraic multiplicity of the eigenvalue $\lambda_{i}$ of $\mathbf{S}^{+}$-matrix (see Ths. 1 and 2). Then for any $i$ and $j$ function $\varphi_{i, j}^{-}(x)$ is normalizable (non-normalizable) at $+\infty$ if and only if $\varphi_{i, k_{i}-j-1}^{+}(x)$ is non-normalizable (normalizable) at $+\infty$. The same takes place at $-\infty$.

Corollary 4. In order that for the level $\lambda_{i}$ the Hamiltonian $h^{+}$does not have eigenfunctions and associated functions normalizable on the whole axis and the Hamiltonian $h^{-}$has a Jordan cell of multiplicity $\nu_{-}\left(\lambda_{i}\right)$ spanned by eigenfunction and associated functions normalizable on whole axis (the same number $\nu_{-}\left(\lambda_{i}\right)$ measures the dimension of the subspace of non-normalizable zero-modes of $\left.q^{-}\right)$it is necessary and sufficient that

$$
k_{i}=\max \left\{k_{i \uparrow}^{+}, k_{i \downarrow}^{+}\right\}+\nu_{-}\left(\lambda_{i}\right)=\left|k_{i \uparrow}^{-}-k_{i \downarrow}^{-}\right|+\nu_{-}\left(\lambda_{i}\right),
$$

where $k_{i \uparrow}^{ \pm}\left(k_{i \downarrow}^{ \pm}\right)$are numbers of functions $\varphi_{i, j}^{\mp}(x)$ normalizable at $+\infty(-\infty)$ (see the previous Sec.). Thus if there are no eigenfunctions and associated functions corresponding to the level lambda $\lambda_{i}$ of the initial Hamiltonian $h^{+}$, normalizable on the whole axis and one wants to get the final Hamiltonian $h^{-}$with a Jordan cell of rank $\nu_{-}\left(\lambda_{i}\right)$ spanned by an eigenfunction and associated functions normalizable on the whole axis, one must choose transformation functions such that they contain $\nu_{-}\left(\lambda_{i}\right)$ (and no more) associated functions of $h^{+}$non-normalizable at both infinities .

A more precise result on interrelation between Jordan structures of intertwined Hamiltonians and on the behavior of transformation functions is given in the

Index Theorem 3: on relation between Jordan structures of intertwined Hamiltonians Let us assume that: 1) conditions of lemma 4 take place; 2) $\nu_{ \pm}(\lambda)$ is the algebraic multiplicity of an eigenvalue $\lambda$ of $h^{ \pm}$, i.e. the number of independent eigenfunctions and associated functions of $h^{ \pm}$normalizable on the whole axis ; 3) if $\lambda=\lambda_{i}$, where $\lambda_{i}$ is an eigenvalue of $\mathbf{S}^{ \pm}$(see Th. 1), then $n_{ \pm}\left(\lambda_{i}\right)$ is a number of normalizable functions at both infinities among $\varphi_{i j}^{\mp}(x), j=0, \ldots, k_{i}-1$ and $n_{0}\left(\lambda_{i}\right)$ is a number of functions normalizable 
only at one of infinities, among $\varphi_{i j}^{\mp}(x), j=0, \ldots, k_{i}-1$. Then the equality

$$
\nu_{+}\left(\lambda_{i}\right)-n_{+}\left(\lambda_{i}\right)=\nu_{-}\left(\lambda_{i}\right)-n_{-}\left(\lambda_{i}\right)
$$

takes place for any $i$. Moreover if $n_{0}(\lambda)>0$ for some $\lambda=\lambda_{j}$, then for this $\lambda_{j}$

$$
\nu_{+}\left(\lambda_{j}\right)-n_{+}\left(\lambda_{j}\right)=\nu_{-}\left(\lambda_{j}\right)-n_{-}\left(\lambda_{j}\right)=0
$$

and if $\lambda$ is not an eigenvalue of $\mathbf{S}^{ \pm}$but $\lambda \leq 0$ or $\operatorname{Im} \lambda \neq 0$, then $\nu_{+}(\lambda)=\nu_{-}(\lambda)$.

\section{An example of non-diagonalizable Hamiltoni- ans made by SUSY transformations}

\subsection{SUSY system with Jordan cell of rank 2}

Let us start from the Darboux transformation of the free particle Hamiltonian (which is trivially PT-symmetric),

$$
h^{+}=-\partial^{2}
$$

and build an isospectral Hamiltonian (its SUSY partner) which is reflectionless [80, 85, 87, due to spectral equivalence to a free particle system,

$$
\begin{gathered}
h^{-}=-\partial^{2}-16 \alpha^{2} \frac{\alpha(x-z) \operatorname{sh}\left(2 \alpha\left(x-x_{0}\right)\right)-2 \operatorname{ch}^{2}\left(\alpha\left(x-x_{0}\right)\right)}{\left[\operatorname{sh}\left(2 \alpha\left(x-x_{0}\right)\right)+2 \alpha(x-z)\right]^{2}}, \\
\alpha>0, \quad x_{0} \in \mathbb{R}, \quad z \in \mathbb{C}, \quad \operatorname{Im} z \neq 0
\end{gathered}
$$

with the help of the intertwining operators $q_{2}^{ \pm}$:

$$
\begin{gathered}
q_{2}^{ \pm} h^{\mp}=h^{ \pm} q_{2}^{ \pm}, \quad q_{2}^{-}=\partial^{2}-\frac{W^{\prime}(x)}{W(x)} \partial-\alpha^{2}+\frac{1}{2} \frac{W^{\prime \prime}(x)}{W(x)}, \quad q_{2}^{+}=\left(q_{2}^{-}\right)^{t}, \\
W(x)=\operatorname{sh}\left(2 \alpha\left(x-x_{0}\right)\right)+2 \alpha(x-z) .
\end{gathered}
$$

If $x_{0}=\operatorname{Re} z=0$ the Hamiltonian $h^{-}$reveals PT-symmetry. Otherwise PT-symmetry is not realized although the energy spectrum remains real.

The operator $q_{2}^{-}$can be factorized into two intertwining operators of first order in derivatives,

$$
q_{2}^{-}=q_{b}^{-} q_{a}^{-}, \quad q_{a}^{-}=\partial-\alpha \operatorname{th}\left(\alpha\left(x-x_{0}\right)\right), \quad q_{b}^{-}=\partial-\frac{W^{\prime}(x)}{W(x)}+\alpha \operatorname{th}\left(\alpha\left(x-x_{0}\right)\right),
$$

with the intermediate non-singular Hamiltonian of the ladder construction of Sec. 4,

$$
h_{1}=-\partial^{2}-\frac{2 \alpha^{2}}{\operatorname{ch}^{2}\left(\alpha\left(x-x_{0}\right)\right)} .
$$

The canonical basis of $q_{2}^{-}$consists of two non-normalizable functions:

$$
\begin{gathered}
\varphi_{0}^{-}(x)=\operatorname{ch}\left(\alpha\left(x-x_{0}\right)\right), \quad \varphi_{1}^{-}(x)=-\frac{(x-z)}{2 \alpha} \operatorname{sh}\left(\alpha\left(x-x_{0}\right)\right)+\frac{1}{4 \alpha^{2}} \operatorname{ch}\left(\alpha\left(x-x_{0}\right)\right), \\
h^{+} \varphi_{0}^{-}=\lambda_{0} \varphi_{0}^{-}, \quad\left(h^{+}-\lambda_{0}\right) \varphi_{1}^{-}=\varphi_{0}^{-}, \quad \lambda_{0}=-\alpha^{2}, \quad \mathbf{S}^{+}=\left(\begin{array}{cc}
\lambda_{0} & 0 \\
1 & \lambda_{0}
\end{array}\right) .
\end{gathered}
$$


On the other hand the canonical basis of $q_{2}^{+}$consists of two normalizable functions:

$$
\varphi_{0}^{+}(x)=(2 \alpha)^{3 / 2} \frac{\varphi_{0}^{-}(x)}{W(x)}, \quad \varphi_{1}^{+}(x)=-(2 \alpha)^{3 / 2} \frac{\varphi_{1}^{-}(x)}{W(x)}
$$

which form the Jordan cell for $h^{-}$corresponding to the level $\lambda_{0}$ :

$$
h^{-} \varphi_{0}^{+}=\lambda_{0} \varphi_{0}^{+}, \quad\left(h^{-}-\lambda_{0}\right) \varphi_{1}^{+}=\varphi_{0}^{+}, \quad \mathbf{S}^{-}=\left(\begin{array}{cc}
\lambda_{0} & 0 \\
1 & \lambda_{0}
\end{array}\right)
$$

In relation to factorization (68) one can show that the zero-mode of $q_{b}^{-}$becomes:

$$
q_{a}^{-} \varphi_{1}^{-}=-\frac{\operatorname{sh}\left(\alpha\left(x-x_{0}\right)\right)}{2 \alpha}-\frac{(x-z)}{2 \operatorname{ch}\left(\alpha\left(x-x_{0}\right)\right)} \equiv-\frac{W(x)}{4 \alpha \operatorname{ch}\left(\alpha\left(x-x_{0}\right)\right)} \equiv-\sqrt{\frac{\alpha}{2}} \frac{1}{\varphi_{0}^{+}} .
$$

In turn the eigenfunctions of $h^{-}$for continuous spectrum read:

$$
\begin{aligned}
& \psi(x ; k)=-\frac{1}{\sqrt{2 \pi}\left(\alpha^{2}+k^{2}\right)} q_{2}^{-} e^{i k x}=\frac{1}{\sqrt{2 \pi}}\left[1+\frac{i k}{\alpha^{2}+k^{2}} \frac{W^{\prime}(x)}{W(x)}-\frac{1}{2\left(\alpha^{2}+k^{2}\right)} \frac{W^{\prime \prime}(x)}{W(x)}\right] e^{i k x}, \\
& k \in \mathbb{R}, \quad h^{-} \psi(x ; k)=k^{2} \psi(x ; k) .
\end{aligned}
$$

One can check that eigenfunctions and associated functions of $h^{-}$obey the relations:

$$
\begin{gathered}
\int_{-\infty}^{+\infty}\left(\varphi_{0,1}^{+}(x)\right)^{2} d x=0, \int_{-\infty}^{+\infty} \varphi_{0}^{+}(x) \varphi_{1}^{+}(x) d x=1, \quad \int_{-\infty}^{+\infty} \varphi_{0,1}^{+}(x) \psi(x ; k) d x=0, \\
\int_{-\infty}^{+\infty} \psi(x ; k) \psi\left(x ;-k^{\prime}\right) d x=\delta\left(k-k^{\prime}\right),
\end{gathered}
$$

where the last relation is understood, as usual, in the sense of distributions.

One can also find that the functions $\varphi_{0}^{+}(x), \varphi_{1}^{+}(x)$ can be obtained by analytical continuation of $\psi(x ; k)$ in $k$,

$$
\begin{gathered}
\lim _{k \rightarrow \pm i \alpha}\left[\left(k^{2}+\alpha^{2}\right) \psi(x ; k)\right]=\mp \sqrt{\frac{\alpha}{\pi}} e^{\mp \alpha x_{0}} \varphi_{0}^{+}(x), \\
\lim _{k \rightarrow \pm i \alpha}\left[\frac{1}{2 k} \frac{\partial}{\partial k}\left(\left(k^{2}+\alpha^{2}\right) \psi(x ; k)\right)\right]=\mp \sqrt{\frac{\alpha}{\pi}} e^{\mp \alpha x_{0}}\left[\varphi_{1}^{+}(x)-\frac{1 \mp 2 \alpha z}{4 \alpha^{2}} \varphi_{0}^{+}(x)\right] .
\end{gathered}
$$

For this model resolution of identity made of eigenfunctions and associated functions of $h^{-}$can be obtained by the conventional Green function method:

$$
\delta\left(x-x^{\prime}\right)=\int_{-\infty}^{+\infty} \psi(x ; k) \psi\left(x^{\prime} ;-k\right) d k+\varphi_{0}^{+}(x) \varphi_{1}^{+}\left(x^{\prime}\right)+\varphi_{1}^{+}(x) \varphi_{0}^{+}\left(x^{\prime}\right)
$$

or in the operator form,

$$
I=\int_{-\infty}^{+\infty}|\psi, k\rangle\left\langle\tilde{\psi}, k|d k+| \psi_{0}\right\rangle\left\langle\tilde{\psi}_{0}|+| \psi_{1}\right\rangle\left\langle\tilde{\psi}_{1}\right|
$$


where the Dirac notations have been used:

$$
\begin{gathered}
\langle x \mid \psi, k\rangle=\psi(x ; k), \quad\langle x \mid \tilde{\psi}, k\rangle=\psi^{*}(x ;-k), \\
\left\langle x \mid \psi_{0,1}\right\rangle=\varphi_{0,1}^{+}(x), \quad\left\langle x \mid \tilde{\psi}_{0,1}\right\rangle=\left(\varphi_{1,0}^{+}(x)\right)^{*}, \\
h^{-\dagger}\left|\tilde{\psi}_{1}\right\rangle=\lambda_{0}\left|\tilde{\psi}_{1}\right\rangle, \quad\left(h^{-\dagger}-\lambda_{0}\right)\left|\tilde{\psi}_{0}\right\rangle=\left|\tilde{\psi}_{1}\right\rangle, \quad h^{-\dagger}|\tilde{\psi}, k\rangle=k^{2}|\tilde{\psi}, k\rangle
\end{gathered}
$$

We stress that $\left|\tilde{\psi}_{0,1}\right\rangle$ are analogs of $\left|\tilde{\psi}_{n, a, i}\right\rangle$ from Sec. 2. In this notations the biorthogonal relations take the form,

$$
\left\langle\tilde{\psi}_{j} \mid \psi_{k}\right\rangle=\delta_{j k}, \quad\left\langle\tilde{\psi}, k \mid \psi_{0,1}\right\rangle=\left\langle\tilde{\psi}_{1,0} \mid \psi, k\right\rangle=0, \quad\left\langle\tilde{\psi}, k \mid \psi, k^{\prime}\right\rangle=\delta\left(k-k^{\prime}\right) .
$$

Accordingly, the spectral decomposition of $h^{-}$can be easily derived,

$$
h^{-}=\int_{-\infty}^{+\infty} k^{2}|\psi, k\rangle\left\langle\tilde{\psi}, k\left|d k-\alpha^{2}\right| \psi_{0}\right\rangle\left\langle\tilde{\psi}_{0}\left|-\alpha^{2}\right| \psi_{1}\right\rangle\left\langle\tilde{\psi}_{1}|+| \psi_{0}\right\rangle\left\langle\tilde{\psi}_{1}\right| .
$$

Let's remind that, for a given $\lambda=\lambda_{i}, k_{i}$ is the number of zero-modes of $q_{2}^{\mp}, k_{i \uparrow, \downarrow}^{ \pm}$ are the numbers of zero-modes normalizable at one of $\pm \infty$ labeled by $\uparrow, \downarrow, \nu_{ \pm}(\lambda)$ are the numbers of eigenfunctions and associated functions of $h^{ \pm}$normalizable on the whole axis, $n_{ \pm}(\lambda)$ are the numbers of eigenfunctions and associated functions among zero-modes $\varphi_{i, j}^{\mp}$ normalizable on the whole axis and $n_{0}(\lambda)$ is the number of eigenfunctions and associated functions among zero-modes $\varphi_{i, j}^{\mp}$ normalizable only at one end. They are defined in Sec. 5, 6 . For this model they take the following particular values $(i=0)$ :

$$
\begin{gathered}
k_{0}=2, \quad k_{0 \uparrow, \downarrow}^{+}=0, \quad \nu_{+}(\lambda) \equiv 0, \quad k_{0 \uparrow, \downarrow}^{-}=2, \quad \nu_{-}(\lambda)= \begin{cases}2, & \lambda=\lambda_{0}, \\
0, & \lambda \neq \lambda_{0},\end{cases} \\
n_{+}(\lambda) \equiv 0 ; \quad n_{0}(\lambda) \equiv 0, \quad n_{-}(\lambda)= \begin{cases}2, & \lambda=\lambda_{0}, \\
0, & \lambda \neq \lambda_{0},\end{cases}
\end{gathered}
$$

and the Index Theorem holds,

$$
\nu_{+}\left(\lambda_{j}\right)-n_{+}\left(\lambda_{j}\right)=\nu_{-}\left(\lambda_{j}\right)-n_{-}\left(\lambda_{j}\right)=0
$$

\subsection{Coalescence of two levels}

The Hamiltonian $h^{-}$with Jordan cell for bound state (66) is a particular limiting case of the Hamiltonian $h^{-}$with two non-degenerate bound states (of algebraic multiplicity 1 ). The former corresponds to the confluent case of the latter one. One again starts from the free particle Hamiltonian,

$$
h^{+}=-\partial^{2}
$$

and obtains its SUSY partner

$$
\begin{gathered}
h^{-}=-\partial^{2}-16 \alpha^{2} \times \\
\frac{\frac{\alpha^{2}+\beta^{2}}{2 \alpha \beta} \operatorname{sh}\left(2 \alpha\left(x-x_{0}\right)\right) \operatorname{sh}(2 \beta(x-z))-2 \operatorname{ch}^{2}\left(\alpha\left(x-x_{0}\right)\right) \operatorname{ch}(2 \beta(x-z))+2 \operatorname{sh}^{2}(\beta(x-z))}{\left[\operatorname{sh}\left(2 \alpha\left(x-x_{0}\right)\right)+\frac{\alpha}{\beta} \operatorname{sh}(2 \beta(x-z))\right]^{2}}
\end{gathered}
$$


by intertwining with the operators

$$
q_{2}^{-}=\partial^{2}-\frac{W^{\prime}(x)}{W(x)} \partial-\left(\alpha^{2}+\beta^{2}\right)+\frac{1}{2} \frac{W^{\prime \prime}(x)}{W(x)}=\left(q_{2}^{+}\right)^{t}
$$

where

$$
\begin{gathered}
W(x)=\operatorname{sh}\left(2 \alpha\left(x-x_{0}\right)\right)+\frac{\alpha}{\beta} \operatorname{sh}(2 \beta(x-z)), \\
x_{0} \in \mathbb{R}, \quad \operatorname{Im} z \neq 0, \quad \alpha>0 \quad(\text { or }-i \alpha>0), \quad 0 \leq \beta<\frac{\pi}{2 \operatorname{Im} z} .
\end{gathered}
$$

In the case $\beta \neq 0, \beta \neq \alpha$ the canonical basis of $q_{2}^{-}$zero-modes (transformation functions) consists of

$$
\varphi_{ \pm \beta}(x)=\operatorname{ch}\left(k_{ \pm \beta}\left(x-\xi_{ \pm \beta}\right)\right), \quad k_{ \pm \beta}=\alpha \pm \beta, \quad \xi_{ \pm \beta}=\frac{\alpha x_{0} \pm \beta z}{\alpha \pm \beta}
$$

so that

$$
h^{+} \varphi_{ \pm \beta}=\lambda_{ \pm \beta} \varphi_{ \pm \beta}, \quad \lambda_{ \pm \beta}=-k_{ \pm \beta}^{2}=-(\alpha \pm \beta)^{2} .
$$

One can check that in the case $\beta \neq 0$ the function $W(x)$ is a Wronskian of $\varphi_{+\beta}(x)$ and $\varphi_{-\beta}(x)$ divided by $\beta$ and in the case $\beta=0$ it is a product of $-4 \alpha$ and of the Wronskian of $\varphi_{0}^{-}(x)$ and $\varphi_{1}^{-}(x)$.

For $\beta=0$ both functions $\varphi_{ \pm \beta}(x)$ coincide with $\varphi_{0}^{-}(x)$ from (70), and as well $\lambda_{ \pm \beta}=\lambda_{0}$. In this case $\varphi_{1}^{-}(x)$ is a linear combination of $\varphi_{+\beta}(x)$ and $\varphi_{-\beta}(x)$ in the following sense,

$$
\varphi_{1}^{-}=\left.\frac{\frac{\partial}{\partial \beta}\left(\varphi_{+\beta}-\varphi_{-\beta}\right)}{\frac{\partial}{\partial \beta}\left(\lambda_{+\beta}-\lambda_{-\beta}\right)}\right|_{\beta=0}+\frac{1}{4 \alpha^{2}} \varphi_{0}^{-} .
$$

In the case $\beta \neq 0$ the canonical basis of zero-modes of $q_{2}^{+}$consists of

$$
\tilde{\psi}_{ \pm \beta}(x)=\frac{\varphi_{\mp \beta}(x)}{W(x)} .
$$

One can prove that

$$
\int_{-\infty}^{+\infty} \tilde{\psi}_{ \pm \beta}^{2}(x) d x=\mp \frac{\beta}{2 \alpha(\alpha \pm \beta)}
$$

(this formula is valid also in the case $\beta=0$ ). The fact that the integrals (84) vanish for $\beta=0$ is in line with (76). It follows from (84) that the normalized eigenfunctions of $h^{-}$ have the form

$\psi_{+\beta}(x)=\sqrt{2} i \alpha \sqrt{\frac{1}{\beta}+\frac{1}{\alpha}} \frac{\varphi_{-\beta}(x)}{W(x)}, \quad \psi_{-\beta}(x)=\sqrt{2} \alpha \sqrt{\frac{1}{\beta}-\frac{1}{\alpha}} \frac{\varphi_{+\beta}(x)}{W(x)}, \quad h^{-} \psi_{ \pm \beta}=\lambda_{ \pm \beta} \psi_{ \pm \beta}$.

The eigenfunctions of $h^{-}$for continuous spectrum read,

$$
\begin{aligned}
& \psi(x ; k)=\frac{\left[\alpha^{2}+\beta^{2}+k^{2}+i k \frac{W^{\prime}(x)}{W(x)}-\frac{1}{2} \frac{W^{\prime \prime}(x)}{W(x)}\right] e^{i k x}}{\sqrt{2 \pi} \sqrt{\left(k^{2}+\alpha^{2}+\beta^{2}\right)^{2}-4 \alpha^{2} \beta^{2}}}, \\
& k \in \mathbb{R}, \quad h^{-} \psi(x ; k)=k^{2} \psi(x ; k),
\end{aligned}
$$

where the branch of $\sqrt{\left(k^{2}+\alpha^{2}+\beta^{2}\right)^{2}-4 \alpha^{2} \beta^{2}}$ can be defined by the condition

$$
\sqrt{\left(k^{2}+\alpha^{2}+\beta^{2}\right)^{2}-4 \alpha^{2} \beta^{2}}=k^{2}+o\left(k^{2}\right), \quad k \rightarrow \infty
$$


in the plane with cuts, linking branch points, situated in the upper (lower) half-plane.

One can prove the following limits,

$$
\begin{aligned}
& \lim _{k \rightarrow \pm i(\alpha+\beta)}\left[\sqrt{\left(k^{2}+\alpha^{2}+\beta^{2}\right)^{2}-4 \alpha^{2} \beta^{2}} \psi(x ; k)\right]= \pm \frac{2 i \alpha \beta}{\sqrt{\pi}} \sqrt{\frac{1}{\beta}+\frac{1}{\alpha}} e^{\mp\left(\alpha x_{0}+\beta z\right)} \psi_{+\beta}(x), \\
& \lim _{k \rightarrow \pm i(\alpha-\beta)}\left[\sqrt{\left(k^{2}+\alpha^{2}+\beta^{2}\right)^{2}-4 \alpha^{2} \beta^{2}} \psi(x ; k)\right]=\mp \frac{2 \alpha \beta}{\sqrt{\pi}} \sqrt{\frac{1}{\beta}-\frac{1}{\alpha}} e^{\mp\left(\alpha x_{0}-\beta z\right)} \psi_{-\beta}(x) .
\end{aligned}
$$

In the limiting case $\beta=0$ the eigenfunction $\varphi_{0}^{+}(x)$ and the associated function $\varphi_{1}^{+}(x)$ of $h^{-}$(see Eqs.(72) ) can be derived from $\psi_{ \pm \beta}(x)$,

$$
\begin{gathered}
\varphi_{0}^{+}(x)=-2 i \sqrt{\alpha} \lim _{\beta \rightarrow 0}\left[\sqrt{\beta} \psi_{+\beta}(x)\right]=2 \sqrt{\alpha} \lim _{\beta \rightarrow 0}\left[\sqrt{\beta} \psi_{-\beta}(x)\right], \\
\varphi_{1}^{+}(x)=2 \sqrt{\alpha} \lim _{\beta \rightarrow 0} \frac{\frac{\partial}{\partial \beta}\left[\sqrt{\beta}\left(\psi_{-\beta}(x)+i \psi_{+\beta}(x)\right)\right]}{\frac{\partial}{\partial \beta}\left(\lambda_{+\beta}-\lambda_{-\beta}\right)} .
\end{gathered}
$$

Resolution of identity in the case $\beta \neq 0$ takes the form:

$$
\delta\left(x-x^{\prime}\right)=\psi_{+\beta}(x) \psi_{+\beta}\left(x^{\prime}\right)+\psi_{-\beta}(x) \psi_{-\beta}\left(x^{\prime}\right)+\int_{-\infty}^{+\infty} \psi(x ; k) \psi(x ;-k) d k
$$

and one can show that in the case $\alpha>0$

$$
\lim _{\beta \rightarrow 0}\left[\psi_{+\beta}(x) \psi_{+\beta}\left(x^{\prime}\right)+\psi_{-\beta}(x) \psi_{-\beta}\left(x^{\prime}\right)\right]=\varphi_{0}^{+}(x) \varphi_{1}^{+}\left(x^{\prime}\right)+\varphi_{1}^{+}(x) \varphi_{0}^{+}\left(x^{\prime}\right)
$$

(cf. with (77)).

One can also check that the biorthogonal relations take place:

$$
\int_{-\infty}^{+\infty} \psi_{+\beta}(x) \psi_{-\beta}(x) d x=0, \quad \int_{-\infty}^{+\infty} \psi_{ \pm \beta}(x) \psi(x ; k) d x=0, \quad \int_{-\infty}^{+\infty} \psi(x ; k) \psi\left(x ;-k^{\prime}\right) d x=\delta\left(k-k^{\prime}\right) .
$$

\subsection{Symmetry operators}

For the Hamiltonian $h^{-}$of Subsec. 1 and 2 there exists the antisymmetric symmetry operator of the fifth order

$$
R_{5}=q_{2}^{-} \partial q_{2}^{+}, \quad R_{5} h^{-}=h^{-} R_{5}, \quad R_{5}^{t}=-R_{5} .
$$

In the case of PT-symmetry, $x_{0}=\operatorname{Re} z=0$ it anticommutes with PT-reflection $\theta_{P} T$, $R_{5} \theta_{P} T=-\theta_{P} T R_{5}$.

The wave function $\varphi_{0}^{+}(x)$, the associated function $\varphi_{1}^{+}(x)$ and the wave function of zero-energy bound state

$$
\psi(x ; 0)=\frac{1}{\sqrt{2 \pi}}\left[1-\frac{1}{2 \alpha^{2}} \frac{W^{\prime \prime}(x)}{W(x)}\right]
$$


are zero-modes of $R_{5}$ because of $\varphi_{0,1}^{+}(x)$ are zero-modes of $q_{2}^{+}$and $q_{2}^{+} \psi(x ; 0)=$ Const. In Subsec. 2 (in the case $\beta \neq 0$ ) the wave functions $\psi_{+\beta}(x), \psi_{-\beta}(x)$ and the wave function of zero-energy bound state

$$
\psi(x ; 0)=\frac{\left[\alpha^{2}+\beta^{2}-\frac{1}{2} \frac{W^{\prime \prime}(x)}{W(x)}\right]}{\sqrt{2 \pi}\left(\alpha^{2}-\beta^{2}\right)}
$$

are zero-modes of $R_{5}$ because of $\psi_{ \pm \beta}(x)$ are zero-modes of $q_{2}^{+}$and $q_{2}^{+} \psi(x ; 0)=$ Const. In both cases the spectrum of $\mathbf{S}$-matrix of the symmetry operator (an analogue of $\mathbf{S}^{+}$from Th. 1) consists of 0 and $-(\alpha \pm \beta)^{2}$.

We notice that if $k \neq 0$ then $\psi(x ; k)$ and $\psi(x ;-k)$ are linearly independent wave functions for the energy level $E=k^{2}$. Simultaneously, $\psi(x ; k)$ is an eigenfunction of the symmetry operator:

$$
R_{5} \psi(x ; k)=i k\left(k^{2}+\alpha^{2}\right)^{2} \psi(x ; k)
$$

or

$$
R_{5} \psi(x ; k)=i k\left[\left(k^{2}+\alpha^{2}+\beta^{2}\right)^{2}-4 \alpha^{2} \beta^{2}\right] \psi(x ; k),
$$

Thus the zeroes of the eigenvalue of $R_{5}$ are related to eigenvalues of $\mathbf{S}$-matrix of $R_{5}$ in accordance to [85].

\section{Conclusions and perspectives: peculiarities of non-Hermitian Hamiltonians with continuous spec- trum}

In our paper we have investigated a bound state part of the spectrum for the class of potentials among which one can find also those ones with continuum spectrum. The choice of this class has allowed to keep all the analysis well below a possible continuum threshold. For this part of the spectrum the relationship between Jordan cells of SUSY partner Hamiltonians is firmly controlled by the Index Theorem 3 and it was well illuminated by an exactly solvable system with two coalescing bound states. We remind that the rigorous proofs of all new Lemmas and the Theorem 3 are postponed to the second part of this paper [65].

Meantime the approaching to the continuum threshold yields more subtle problems with normalizable eigen- and associated functions in continuum which may have zero binorm. As a consequence it may cause serious problems with the resolution of identity - This interesting problem we investigated in 32. Here we would like only to draw attention to a class of models where the continuous spectrum is involved and elaborate the resolution of identity.

Let us consider the model Hamiltonians

$$
h^{+}=-\partial^{2}, \quad h^{-}=-\partial^{2}+\frac{2}{(x-z)^{2}}, \quad \operatorname{Im} z \neq 0,
$$

intertwined by the first-order operators $q_{1}^{ \pm}$:

$$
h^{ \pm} q_{1}^{ \pm}=q_{1}^{ \pm} h^{\mp}, \quad q_{1}^{ \pm}=\mp \partial-\frac{1}{x-z} .
$$

If $\operatorname{Re} z=0$ these Hamiltonians are PT-symmetric and respectively their eigenfunctions possess definite PT- parities. 
The eigenfunctions of $h^{-}$of continuous spectrum can be found in the form,

$$
\psi(x ; k)=\frac{1}{\sqrt{2 \pi}}\left[1-\frac{1}{i k(x-z)}\right] e^{i k x}, \quad k \in \mathbb{R} \backslash\{0\}, \quad h^{-} \psi(x ; k)=k^{2} \psi(x ; k) .
$$

In addition, there is a normalizable eigenfunction of $h^{-}$on the lower end of continuous spectrum:

$$
\psi_{0}(x)=\frac{1}{(x-z)}=-\sqrt{2 \pi} \lim _{k \rightarrow 0}[i k \psi(x ; k)], \quad h^{-} \psi_{0}=0 .
$$

Isospectral relations between $h^{+}$and $h^{-}$take the form,

$$
\begin{gathered}
q^{-}\left[\frac{e^{i k x}}{\sqrt{2 \pi}}\right]=i k \psi(x ; k), \quad k \neq 0, \quad q^{-}\left[\frac{1}{\sqrt{2 \pi}}\right]=\frac{-1}{\sqrt{2 \pi}} \psi_{0}(x), \quad k=0 \\
q^{+} \psi(x ; k)=-i k\left[\frac{e^{i k x}}{\sqrt{2 \pi}}\right], \quad k \neq 0, \quad q^{+} \psi_{0}=0, \quad k=0 .
\end{gathered}
$$

The eigenfunctions of $h^{-}$satisfy the relations of biorthogonality,

$$
\int_{-\infty}^{+\infty} \psi_{0}^{2}(x) d x=0, \quad \int_{-\infty}^{+\infty} \psi_{0}(x) \psi(x ; k) d x=0
$$

Resolution of identity made of eigenfunctions of $h^{-}$can be built as follows,

$$
\delta\left(x-x^{\prime}\right)=\int_{\mathcal{L}} \psi(x ; k) \psi\left(x^{\prime} ;-k\right) d k,
$$

where the contour $\mathcal{L}$ must be a proper integration path in the complex $k$ plane which allows to regularize the singularity in (90) for $k=0$ circumventing it from up or from down. To reach an adequate definition of resolution of identity one can instead use the Newton-Leibnitz formula and rewrite (95) in the form

$$
\begin{gathered}
\delta\left(x-x^{\prime}\right)=\left(\int_{-\infty}^{-\varepsilon}+\int_{\varepsilon}^{+\infty}\right) \psi(x ; k) \psi\left(x^{\prime} ;-k\right) d k \\
-\frac{\psi_{0}(x) \psi_{0}\left(x^{\prime}\right)}{\pi \varepsilon}+\frac{\sin \varepsilon\left(x-x^{\prime}\right)}{\pi\left(x-x^{\prime}\right)}+\frac{2 \sin ^{2}\left(\frac{\varepsilon}{2}\left(x-x^{\prime}\right)\right)}{\pi \varepsilon(x-z)\left(x^{\prime}-z\right)}, \quad \varepsilon>0 .
\end{gathered}
$$

One can show [32] that the limit of the 3rd term of the right side of (96) (as a distribution) under $\varepsilon \downarrow 0$ is zero for any test function from $C_{\mathbb{R}}^{\infty} \cap L^{2}(\mathbb{R})$ but the limit of the last term of the right side of (96) under $\varepsilon \downarrow 0$ is zero only for test functions from $C_{\mathbb{R}}^{\infty} \cap L^{2}\left(\mathbb{R} ;|x|^{\gamma}\right)$, $\gamma>1$. Thus for test functions from $C_{\mathbb{R}}^{\infty} \cap L^{2}\left(\mathbb{R} ;|x|^{\gamma}\right), \gamma>1$ resolution of identity can be reduced to,

$$
\delta\left(x-x^{\prime}\right)=\lim _{\varepsilon \downarrow 0}\left[\left(\int_{-\infty}^{-\varepsilon}+\int_{\varepsilon}^{+\infty}\right) \psi(x ; k) \psi\left(x^{\prime} ;-k\right) d k-\frac{\psi_{0}(x) \psi_{0}\left(x^{\prime}\right)}{\pi \varepsilon}\right]
$$


and for test functions from $C_{\mathbb{R}}^{\infty} \cap L^{2}(\mathbb{R})$ to,

$$
\begin{aligned}
\delta\left(x-x^{\prime}\right)= & \lim _{\varepsilon \downarrow 0}\left\{\left(\int_{-\infty}^{-\varepsilon}+\int_{\varepsilon}^{+\infty}\right) \psi(x ; k) \psi\left(x^{\prime} ;-k\right) d k\right. \\
& \left.-\frac{1}{\pi \varepsilon}\left[1-2 \sin ^{2}\left(\frac{\varepsilon}{2}\left(x-x^{\prime}\right)\right)\right] \psi_{0}(x) \psi_{0}\left(x^{\prime}\right)\right\} .
\end{aligned}
$$

Decomposition (97) seems to have a more natural form than (98), but its right side obviously cannot reproduce the normalizable eigenfunction

$$
\psi_{0}(x) \notin C_{\mathbb{R}}^{\infty} \cap L^{2}\left(\mathbb{R} ;|x|^{\gamma}\right), \quad \gamma>1
$$

because of the orthogonality relations (94). One can show that

$$
\lim _{\varepsilon \downarrow 0} \int_{-\infty}^{+\infty} \frac{2}{\pi \varepsilon} \sin ^{2}\left(\frac{\varepsilon}{2}\left(x-x^{\prime}\right)\right) \psi_{0}^{2}(x) \psi_{0}\left(x^{\prime}\right) d x=\lim _{\varepsilon \downarrow 0}\left[e^{-i \varepsilon x^{\prime}} \psi_{0}\left(x^{\prime}\right)\right]=\psi_{0}\left(x^{\prime}\right) .
$$

Thus it is the $3 \mathrm{rd}$ term in the right side of (98) that provides the opportunity to reproduce $\psi_{0}(x)$ and thereby to complete the resolution of identity.

The spectral decomposition of $h^{-}$in this case reads

$$
h^{-}=\int_{-\infty}^{+\infty} k^{2}|\psi, k\rangle\langle\tilde{\psi}, k| d k
$$

where

$$
\langle x \mid \psi, k\rangle=\psi(x ; k), \quad\langle x \mid \tilde{\psi}, k\rangle=\psi^{*}(x ;-k), \quad h^{-\dagger}|\tilde{\psi}, k\rangle=k^{2}|\tilde{\psi}, k\rangle .
$$

For the Hamiltonian $h^{-}$of this model there is an antisymmetric symmetry operator of the 3rd order

$$
\begin{gathered}
R_{3}=q_{1}^{-} \partial q_{1}^{+}=-\partial^{3}+\frac{3}{(x-z)^{2}} \partial-\frac{3}{(x-z)^{3}}, \\
R_{3} h^{-}=h^{-} R_{3}, \quad R_{3}^{t}=-R_{3}, \quad R_{3} \psi(x ; k)=i k^{3} \psi(x ; k) .
\end{gathered}
$$

We notice that this model is a limiting case of the example of Subsec. 7.1 for $\alpha \rightarrow 0$ where $z$ must be taken as a half sum of $x_{0}$ and $z$.

One can generalize this model constructing the Hamiltonian (by intertwining with the Hamiltonian of a free particle)

$$
h^{-}=-\partial^{2}+\frac{n(n+1)}{(x-z)^{2}}
$$

for which there is a Jordan cell, spanned by $\left[\frac{n+1}{2}\right]$ normalizable eigenfunction and associated functions:

$h \psi_{0}=0, \quad h \psi_{j}=\psi_{j-1}, \quad j=0, \ldots,\left[\frac{n-1}{2}\right], \quad \psi_{j}(x)=\frac{(2(n-j)-1) ! !}{(2 j) ! !(2 n-1) ! !(x-z)^{n-2 j}}$,

at the bottom of continuous spectrum . All these functions are mutually biorthogonal having also zero binorm. The problem of a correct resolution of identity seems to be solvable in the same way as for $n=1$. However the rigorous analysis is postponed to a forthcoming paper. 


\section{Acknowledgements}

The work of A.A. and A.S. was supported by Grant RFBR 06-01-00186-a and by Programs RNP 2.1.1.1112 and LSS-5538.2006.2. A.Sokolov was partially supported by the INFN grant.

\section{References}

[1] H. Feshbach, Theoretical Nuclear Theory: Nuclear Reactions, Wiley, New York, 1992;

P. E. Hodgson, The Nucleon Optical Potential, World Scientific, Singapore, 1995 .

[2] C. Itzykson, J.-M. Drouffe, Statistical Field Theory, V.1, Cambridge University Press, Cambridge, 1989 .

[3] N. Moiseyev, Phys. Rep. 302 (1998) 211.

[4] J. G. Muga, J. P. Palao, B. Navarro, I. L. Egusquiza, Phys. Rep. 395 (2004) 357.

[5] N. Dunford, Bull. Amer. Math. Soc. 64 (1958) 217.

[6] B. S. Pavlov, Itogi Nauki i Tekhniki, Sovrem. Probl. Mat. Fund. Naprav. 65 (1991) 95; Encyclopaedia Math. Sci. 65 [Partial differential equations, VIII], Springer-Verlag, Berlin, 1996 .

[7] E. B. Davies, Bull. London Math. Soc. 34 (2002) 513 and refs. therein.

[8] S. L. Yakovlev, Theor. Math. Phys. 102 (1995) 323; ibid. 107 (1996) 513.

[9] C. M. Bender, K. A. Milton, Phys. Rev. D 55 (1997) R3255 ;

[10] C. M. Bender, S. Boettcher, Phys. Rev. Lett. 80 (1998) 5243;

C. M. Bender, S. Boettcher, P. Meisinger, J. Math. Phys. 40 (1999) 2201.

[11] C. M. Bender, D. C. Brody, H. F. Jones, Phys.Rev.Lett. 89 (2002) 270401; Erratumibid. 92 (2004) 119902; Am. J. Phys. 71 (2003) 1095;

C. Bender, J. Brod, A. Refig, M. Reuter, J. Phys. A: Math. Gen. 37 (2004) 10139;

C. Bender, quant-ph/0501052;

C. M. Bender, J.-H.Chen, K. A. Milton, J.Phys. A39 (2006) 1657;

C. M. Bender, D. C. Brody, H. F. Jones and B. K. Meister, Phys. Rev. Lett. 98 (2007) 040403;

C. M. Bender, hep-th/0703096.

[12] Z. Ahmed, C. M. Bender, M. V. Berry, Preprint quant-ph/0508117.

[13] M. Znojil, F. Cannata, B. Bagchi, R. Roychoudhury, Phys. Lett. B 483 (2000) 284.

[14] G. Lévai, F. Cannata, A. Ventura, Phys. Lett. A 300 (2002) 271.

[15] A. Mostafazadeh, J. Math. Phys. 43(2002) 205 ; ibid. 43 (2002) 6343 ( A. Mostafazadeh, Erratum-ibid. 44 (2003) 943) .

[16] A. Mostafazadeh, Nucl. Phys. B 640 (2002) 419; J. Phys. A: Math. Gen. 36 (2003) 7081 .

[17] G. Lévai, F. Cannata, A. Ventura, J. Phys. A: Math. Gen. 34 (2001) 839 ;

G. Lévai, F. Cannata, A. Ventura, J. Phys. A: Math. Gen. 35 (2002) 5041 ;

R. N. Deb, F. Khare, B. D. Roy, Phys. Lett. A 307 (2003) 215 ;

A. Mostafazadeh, J. Math. Phys. 46 (2005) 102 . 
[18] F. Cannata, G. Junker, J. Trost, Phys. Lett. A 246 (1998) 219 .

[19] A. A. Andrianov, F. Cannata, J.-P. Dedonder, M. V. Ioffe, Int. J. Mod. Phys. A 14 (1999) 2675 .

[20] T. Tanaka, J. Phys. A: Math. Gen. 39 (2006) 7757; L369;

A. Mostafazadeh, quant-ph/0606173.

[21] A. A. Andrianov, Ann. Phys. 140 (1982) 82 .

[22] V. Buslaev, V. Grecchi, J. Phys. A: Math.Gen. 26 (1993) 5541.

[23] P. Dorey, C. Dunning, R. Tateo, J. Phys. A: Math. Gen. 34 (2001) L391.

[24] H. F. Jones, J. Mateo, Phys. Rev. D 73, 085002 (2006);

C. M. Bender, D. C. Brody and H. F. Jones, Phys. Rev. D 73 (2006) 025002;

C. M. Bender,D. C. Brody, J.-H.Chen, H. F. Jones, K. A. Milton, M. C. Ogilvie, Phys. Rev. D74 (2006) 025016;

H. F. Jones, J. Mateo, R. J. Rivers, Phys. Rev. D 74, 125022 (2006);

H. F. Jones, R. J. Rivers, Phys. Rev. D 75, 025023 (2007) .

[25] T. Curtright, L. Mezincescu, quant-ph/0507015 and references therein .

[26] E. A. Solov'ev, Usp. Fiz. Nauk. 157 (1989) 228 and refs. therein.

[27] M. V. Berry, J. Phys. A: Math. Gen. 31 (1998) 3493.

[28] E. Narevicius, P. Serra, N. Moiseyev, Europhys. Lett. 62 (2003) 789 .

[29] M. Znojil, Phys. Lett. A 259 (1999) 220; ibid. A 264 (1999) 108;

A. Sinha, G. Levai, P. Royc, Phys. Lett. A 322 (2004) 78.

[30] B. F. Samsonov, Pinaki Roy, J. Phys. A: Math. Gen. 38 (2005) L249 .

[31] A. Bohm, M. Loewe, S. Maxson, P. Patuleanu, C. Püntmann, J. Math. Phys.38 (1997) 6072 ;

I. E. Antoniou, M. Gadella, G. P. Pronko, J. Math. Phys. 39 (1998) 2459;

E. Hernández, A. Jáuregui, A. Mondragón, Phys. Rev. A 67 (2003) 022721;

O. Civitarese, M. Gadella, Phys. Rep. 396 (2004) 41 .

[32] A. V. Sokolov , A. A. Andrianov, F. Cannata, J. Phys. A : Math.Gen. 39 (2006) 10207 .

[33] A.A. Andrianov, M.V. Ioffe, V.P. Spiridonov, Phys. Lett. A 174 (1993) 273.

[34] A.A. Andrianov, F. Cannata, J-P. Dedonder, M.V. Ioffe, Int. J. Mod. Phys. A 10 (1995) 2683.

[35] V.G. Bagrov, B.F. Samsonov, Theor. Math. Phys. 104 (1995) 1051.

[36] B.F. Samsonov, Mod. Phys. Lett. A 11 (1996) 1563.

[37] A. Gangopadhyaya, U. Sukhatme, Phys. Lett. A 224 (1996) 5.

[38] U. Sukhatme, C. Rasinariu, A. Khare, Phys. Lett. A 234 (1997) 401.

[39] A. Das, S.A. Pernice, Mod. Phys. Lett. A 12 (1997) 581.

[40] D.J. Fernández C., Int. J. Mod. Phys. A 12 (1997) 171.

[41] G. Junker, P. Roy, Ann. Phys. 270 (1998) 155.

[42] D.J. Fernández C., V. Hussin, B. Mielnik, Phys. Lett. A 244 (1998) 309.

[43] J. O. Rosas-Ortiz, J. Phys. A: Math. Gen.31 (1998) 10163. 
[44] B. Bagchi, A. Ganguly, D. Bhaumik, A. Mitra, Mod. Phys. Lett. A 14 (1999) 27.

[45] D. J. Fernández C., J. Negro, L.M. Nieto, Phys. Lett. A 275 (2000) 338.

[46] D.J. Fernández C., V. Hussin, quant-ph/0011004.

[47] M.S. Plyushchay, Int. J. Mod. Phys. A 15 (2000) 3679; Phys.Lett. B 485 (2000) 187.

[48] H. Aoyama, M. Sato, T. Tanaka, M. Yamamoto, Phys. Lett. B498 (2001) 117.

[49] H. Aoyama, M. Sato, T. Tanaka, Phys. Lett. B 503 (2001) 423.

[50] H. Aoyama, M. Sato, T. Tanaka, Nucl. Phys. B 619 (2001) 105.

[51] H. Aoyama, N. Nakayama, M. Sato, T. Tanaka, Phys. Lett. B 519 (2001) 260; Phys. Lett. B 521 (2001) 400.

[52] T. Tanaka, Nucl. Phys. B 662 (2003) 413.

[53] S.M. Klishevich, M.S. Plyushchay, Nucl. Phys. B 628 (2002) 217.

[54] D. J. Fernández C., R. Muñoz, A. Ramos, quant-ph/0212026.

[55] A.P. Veselov, A.B. Shabat, Funct. Anal. Appl. 27 (1993) 81.

[56] V.E. Adler, Funct. Anal. Appl. 27 (1993) 140.

[57] A.A. Andrianov, M.V. Ioffe, D.N. Nishnianidze, Phys. Lett. A 201 (1995) 103; Theor. Math. Phys. 104 (1995) 1129; J. Phys. A: Math. Gen. 32 (1999) 4641.

[58] A. A. Andrianov, F. Cannata, M.V. Ioffe, D.N. Nishnianidze, J. Phys. A: Math. Gen. 30 (1997) 5037.

[59] F. Cannata, M.V. Ioffe, D.N. Nishnianidze, J. Phys. A: Math. Gen. 35 (2002) 1389.

[60] H. Aoyama, H. Kikuchi, I. Okouchi, M. Sato, S. Wada, Nucl. Phys. B 553 (1999) 644.

[61] B. Bagchi, F. Cannata, C. Quesne, Phys. Lett. A 269 (2000) 79.

[62] R. Sasaki, K. Takasaki, J. Phys. A: Math. Gen. 34 (2001) 9533.

[63] S.M. Klishevich, M.S. Plyushchay, Nucl. Phys. B 606 (2001) 583; Nucl. Phys. B 616 (2001) 403;

[64] P. Dorey, C. Dunning, R. Tateo, J. Phys. A: Math. Gen. 34 (2001) 5679.

[65] A.V.Sokolov, math-ph/0610022 .

[66] N. Moiseyev, S. Friedland, Phys. Rev. A 22 (1980) 618.

[67] S. Weigert, Czech J. Phys. 55 (2005) 1183 and refs. therein.

[68] M. A. Naimark, Linear differential operators. Frederick Ungar Publishing Co., New York, 1967 .

[69] F. R. Gantmacher, The theory of matrices . AMS Chelsea Publishing, Providence, RI, 1998 .

[70] H. Nicolai, J. Phys. A: Math. Gen. 9 (1976) 1497.

[71] E. Witten, Nucl. Phys. B 188 (1981) 513.

[72] F. Cooper, B. Freedman, Ann. Phys. (N.Y.) 146 (1983) 262.

[73] A. A. Andrianov, N. V. Borisov, M. V. Ioffe, JETP Lett. 39 (1984) 93; Phys. Lett. A 105 (1984) 19; Theor. Math. Phys. 61 (1985) 1078. 
[74] M. M. Nieto, Phys. Lett. B 145 (1984) 208.

[75] B. Mielnik, J. Math. Phys. 25 (1984) 3387.

[76] D. Fernández, Lett. Math. Phys. 8 (1984) 337.

[77] A. A. Andrianov, N. V. Borisov, M. V. Ioffe, M. I. Eides, Phys. Lett. A 109 (1985) 143; Theor. Math. Phys. 61 (1985) 965.

[78] C. V. Sukumar, J. Phys. A: Math. Gen. 18 (1985) L57; 2917; 2937.

[79] L. E. Gendenshtein, I. V. Krive, Sov. Phys. Usp. 28 (1985) 645.

[80] A. Lahiri, P. K. Roy, B. Bagchi, Int. J. Mod. Phys. A 5 (1990) 1383.

[81] F. Cooper, A. Khare, U. Sukhatme, Phys. Rept. 251 (1995 )267.

[82] G. Junker, Supersymmetric Methods in Quantum and Statistical Physics . Springer, Berlin-Heidelberg, 1996.

[83] A. A. Andrianov, F. Cannata, J. Phys. A: Math. Gen. 37 (2004) 10297.

[84] B. Mielnik, O. Rosas-Ortiz, J. Phys. A: Math. Gen. 37 (2004) 10007

[85] A. A. Andrianov, A. V. Sokolov, Nucl. Phys. B 660 (2003) 25.

[86] V. B. Matveev, M. A. Salle, Darboux Transformations and Solitons . Springer, BerlinHeidelberg, 1991.

[87] S. P. Maydanyuk, Ann. Phys. (NY) 316 (2) (2005) 440; hep-th/0607125. 